\title{
Using Pareto filters to support risk management in optimization under uncertainty: Application to the strategic planning of chemical supply chain
}

Sergio Medina-Gonzáleza, Carlos Pozoc, Gabriela Corsanod, Gonzalo Guillén-Gósalbez ${ }^{\mathrm{b}, \mathrm{c}}$ and Antonio Espuña ${ }^{a^{*}}$

aChemical Engineering Department, Universitat Politècnica de Catalunya, ETSEIB. Diagonal Avenue 647, 08028 Barcelona, Spain.

b Departament d'Enginyeria Química, Universitat Rovira i Virgili, Av. Països Catalans 26, 43007 Tarragona, Spain c Centre for Process Systems Engineering (CPSE), Imperial College London, SW7 2AZ, United Kingdom dINGAR-Instituto de Desarrollo y diseño (CONICET-UTN), Avellaneda 3657, s3002 GJC Santa Fe, Argentina. *corresponding author: antonio.espuna@upc.edu

\begin{abstract}
Optimization under uncertainty has attracted recently an increasing interest in the process systems engineering literature. The inclusion of uncertainties in an optimization problem inevitably leads to the need to manage the associated risk in order to control the variability of the objective function in the uncertain parameters space. So far, risk management methods have focused on optimizing a single risk metric along with the expected performance. In this work we propose an alternative approach that can handle several risk metrics simultaneously. First, a multi-objective stochastic model containing a set of risk metrics is formulated. This model is then solved efficiently using a tailored decomposition strategy inspired on the Sample Average Approximation. After a normalization step, the resulting solutions are assessed using Pareto filters, which identify solutions showing better performance in the uncertain parameters space. The capabilities and benefits of our approach are illustrated through a design and planning supply chain case study.
\end{abstract}

Keywords: Financial risk metrics, Uncertainty, Multi-objective, and Pareto filters

\section{INTRODUCTION}

Market globalization is continuously producing changes in business behavior, thus making it difficult to predict future trends with certainty. When analyzing the decision-making processes around a typical supply chain (SC), uncertainties, like market demands, raw materials availability, inventory levels and flows, should be considered to capture their direct and local effects over the individual echelons as well as their indirect effects that propagate to other echelons through existing links between them. However, those effects and specially the indirect ones have often been overlooked by the traditional mathematical models used in industry, which are commonly built over the assumption that all the information is known with accuracy beforehand (Zamarripa et al., 2014).

Optimization under uncertainty and, in particular, stochastic programming addresses this challenge by defining recourse actions that allow reacting against every possible realization of the uncertain parameters (Birge and Louveaux, 2011). In this context, a given design might obtain different results depending on the scenario in which it is evaluated, and it is very likely that the optimal design calculated for nominal conditions might render suboptimal (or even unfeasible) under other circumstances. Commonly, stochastic programs are solved over a number of stages, being the twostage stochastic models the most studied ones in Supply Chain Management (SCM) problems. In 
those, stage-1 decisions involve the selection of the design variables for the first time period, whereas stage- 2 decisions are modeled using variables that can be adjusted according to the realization of the scenarios (e.g. recycles in a flowsheet, production levels in a SCM problem)(Grossmann and Guillén-Gosálbez, 2010; Guillén-Gosálbez and Grossmann, 2009,2010; Ben-Tal et al., 2009; Janax et al., 2007). This allows stochastic programming models to react after a scenario materializes (corrective action). As acknowledged by different authors, the main weakness of the traditional stochastic approaches lies in the lack of control on how information regarding uncertain parameters affects optimal decisions. Ierapetritou et al., (1996) emphasized the need of an information index in order to evaluate the quality of the solution associated to the uncertain input data (named Value of perfect information (VPI)). Bernardo et al., $(2000,2001)$ and Ahmed and Sahinidis (1998) proposed a robustness index as a way to evaluate the confidence of the information used, and ultimately provide a robust and confident solution. The robustness index has been applied and evaluated recently in terms of computational effort and solution quality ( $\mathrm{Li}$ and Floudas, 2014), yet, the quality of the predicted information used is out of the scope of the present work.

Standard stochastic approaches tend to optimize the expected performance of the objective function distribution as unique criterion. This strategy provides no control on the variability of the objective function in the uncertain parameters space. One way to overcome such limitation consists of incorporating risk metrics into the model. For instance, Cheng et al. (2003) solved a design and planning uncertainty problem considering multiple objectives, in which one of those objectives was the Downside risk $(D R)$ metric. Additionally, the choice of the appropriate risk metric for the problem at hand is another issue to be considered. Several types of risk metrics have been evaluated in the literature. Barbaro and Bagajewicz (2004) included financial risk management in the framework of two-stage stochastic programming for a planning problem using Financial risk (henceforth known as risk) and $D R$ as risk metrics. On the other hand, Bonfill et al. (2004) and You et al. (2009) used risk, $D R$ and Worst Case (WC) metrics as a way to handle risk management in scheduling and planning problems under uncertainty. More recently, Sabio et al. (2014) minimized separately the $W C$ and $D R$ metrics as a way to reduce the probability of not meeting some environmental targets in the multi-objective optimization (MOO) of industrial networks.

According to Aseeri and Bagajewicz (2004), no single risk metric can be regarded as "complete" risk metric, since they all present at least one of the following disadvantages: lack of associated probability value, limited solution space exploration (i.e., they focus on down, middle or upper side) or lack of capability of assessing simultaneously the probability and potential level of winnings and/or losses. Indeed, in practice most metrics tend to concentrate on penalizing the worst scenarios rather than rewarding the best ones, thereby leading to "risk averse" solutions.

To overcome these limitations, Aseeri and Bagajewicz (2004) proposed a Risk area Ratio (RAR) metric to compare the potential winnings against losses for the entire risk curve using a single value. This metric is useful because it considers the full risk spectrum, yet it does not achieve a simultaneous/complete financial risk analysis. In 2004 Barbaro and Bagajewicz (2004) found a close relation between $D R$ and risk that was used to compute the latter without the need to define binary variables, thereby simplifying the associated calculations. Here it is important to notice that the minimum $D R$ at a defined target profit $(\Omega)$ does not guarantee that risk is minimum at every 
single value of profit $(\leq \Omega)$. Therefore, this relation is an indirect way of measuring financial risk, but not a simultaneous analysis of economic metrics

In summary, there is no single risk metric capable of providing a full control of the objective function in the uncertain parameters space. Hence, ideally, several complementary risk metrics should be optimized along with the expected performance. To the best of our knowledge, however, the simultaneous optimization of several risk metrics has never been addressed in the literature, which constitutes an important gap already acknowledged by several authors (Cheng et al., 2003; Barbaro and Bagajewicz, 2004; Aseeri and Bagajewicz, 2004; Cardoso et al., 2016). One possible reason why this approach has never been applied is that the incorporation of several risk metrics in optimization under uncertainty leads to MOO problems containing a large number of objectives that are difficult to solve for different reasons. First, because generating Pareto solutions of stochastic models with a large number of objectives is computationally challenging. Second, because these stochastic multi-objective models tend to contain an infinite number of Pareto solutions, so even if a representative subset of them is generated, there is still the issue of interpreting and selecting the best solution.

This work proposes a novel approach for optimization under uncertainty and risk management that considers several risk metrics simultaneously during the optimization step. First, a set of solutions behaving in different ways in the uncertain parameters space are generated using an algorithm based on the Sample Average Approximation (SAA) algorithm. Then, the "Pareto filter approach", developed by Mattson et al.,( 2003, 2004) and later used by Pozo et al. (2012b) and Antipova et al. (2015) is applied to rank these solutions. In order to illustrate the capabilities of this approach, the strategic planning problem over a supply chain under uncertainty is used as benchmark. The problem is solved considering different financial risk metrics and identifying strategic decisions that are particularly appealing for decision-makers.

\section{Problem statement}

We address the design of a SC of multi-product batch processes as schematized in Fig. 1. The SC includes a set of raw material suppliers $s p \in N_{s p}$ from which supplier $s p$ can provide one or more types of raw materials $r \in N_{r}$, that are delivered to the batch plants $l \in N_{l}$. Each multiproduct batch plant has a set of batch stages $j \in N_{j l}$, for producing a set of products $i \in N_{i}$. In phase and out of phase unit duplication are considered for each multiproduct batch plant. The use and allocation of intermediate storage tanks is assumed as feasible at each of the $\left|N_{j l}\right|-1$ positions in plant $l$, between two batch stages $(j$ and $j+1)$. Final products are transported from batch plants to different warehouses $m \in N_{m}$, according to their capacity limitation. Products are then delivered from the warehouses to different customer zones $g \in N_{g}$, in order to satisfy a given product demand $D_{i g}$. Further details on this SC design can be found in (Corsano et al., 2011, 2014). 


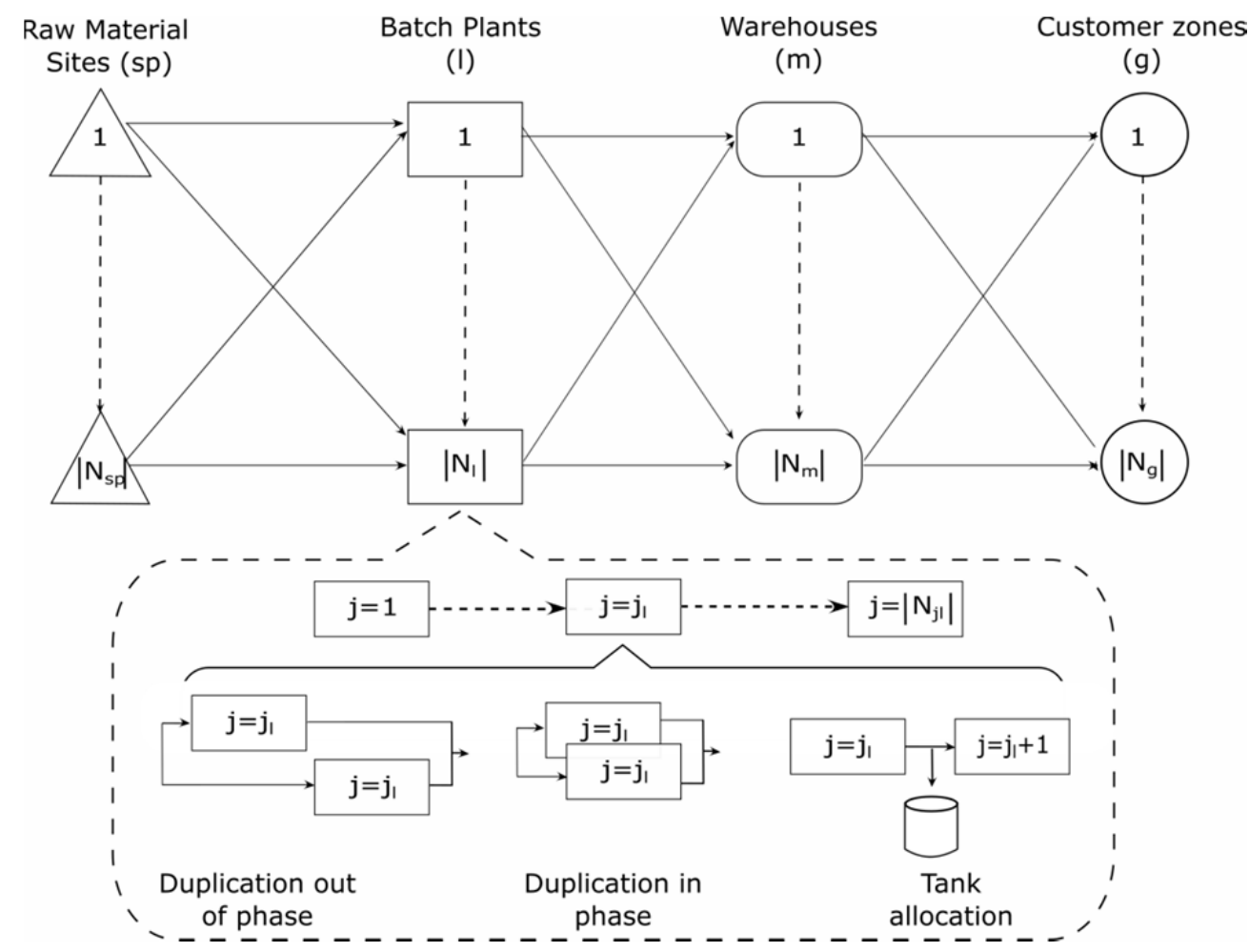

Fig. 1. Process scheme of the supply chain under analysis.

Given are the following data: discrete size of each batch unit to be eventually installed; set of allowable tank sizes and data concerning raw material procurements, distribution cost from-to different sites and overall batch plant parameters. Product demands are modelled as uncertain parameters following known probability distribution patterns whose characteristic parameters are also given. The goal of the analysis is to identify the best planning and design decisions (e.g. number of plants to be installed, equipment units selected, etc.) in terms of maximum expected economic performance and minimum risk.

\section{Methodology}

The proposed approach comprises 4 main steps as shown in Fig. 2. A stochastic MOO model is developed in step 1. Step 2 solves the stochastic MOO problem using a customized strategy that provides as output a set of solutions that are then normalized in step 3. Finally, the normalized solutions are filtered in order to obtain a reduced subset of alternatives with better overall performance. A detailed description of each step is provided in the following subsections. 


\section{Multi-Criteria decision making under uncertainty}

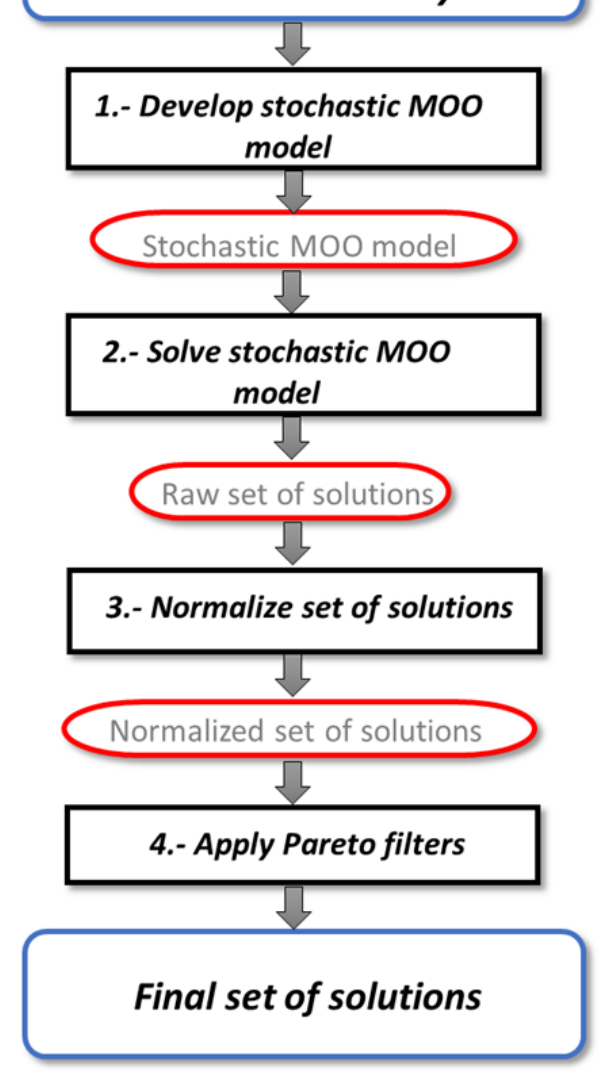

Fig. 2. Overview of the proposed methodology.

\subsection{Multi-scenario two-stage stochastic programming model}

The capabilities of our methodological framework are demonstrated by solving the MILP model presented by Corsano et al. (2014). Hence, the original deterministic single objective (SO) model was reformulated into a multi-scenario two-stage stochastic problem of the following form (see Eq.(1)), henceforth known as Model P:

$$
\max _{x y} f(x, y, \theta)
$$

s.t.

$$
\begin{gathered}
h(x, y, \theta)=0 \\
g(x, y, \theta) \leq 0 \\
x \in \mathrm{X}, y \in \mathrm{Y}, \theta \in \Theta
\end{gathered}
$$

8 Here, $x$ and $y$ are the first and second-stage decision variables, respectively, whereas $\theta$ denotes the

9 uncertain parameters values that belong to the space $\Theta$ of uncertain parameters. First-stage decisions 10 may contain integers due to allocation requirements. $f(x, y, \theta)$ represents the objective function; 
$1 h(x, y, \theta)$ and $g(x, y, \theta)$ are vectors of equality and inequality constraints. Commonly, uncertain 2 parameters are described via scenarios, therefore, model $\mathrm{P}$ can be re-written as follows:

$$
\max _{x, y_{s}} f_{o b}=\sum_{s}^{S} \operatorname{prob}_{s} f\left(x, y_{s}, \theta_{s}\right)
$$

s.t.

$$
\begin{array}{rr}
h\left(x, y_{s}, \theta_{s}\right)=0 & \forall s \in \mathrm{S} \\
g\left(x, y_{s}, \theta_{s}\right) \leq 0 & \forall s \in \mathrm{S} \\
x \in \mathrm{X}, y_{s} \in \mathrm{Y}, \theta_{s} \in \Theta &
\end{array}
$$

Here, $f_{o b}$ represents the expected value for the objective function of the problem P. $\theta_{s}$ is the vector of values taken by the uncertain parameters in the scenarios $s$ and $\operatorname{prob}_{s}$ is the probability of occurrence of scenario s belonging to the set $S$. Model P can be interpreted as follows: First stage decision variables $(x)$ must be taken before a realization of the random vector $\left(\theta_{S}\right)$ becomes known (here and now decisions). However, such a decision needs to satisfy as well the second-stage set of constraints. Therefore, recourse actions need to be taken (second-stage decision variables for each one of the considered scenarios $y_{s}$ ) with an associated impact over the objective function. Hence, given a first-stage decision $x$, each realization of $\theta_{s}$ leads to recourse costs given by the value of the second-stage function $\left(y_{s}\right)$. Note that the parameters values in each scenario can be generated via sampling on the corresponding probability functions.

To manage the risk associated with the decision-making problem under uncertainty, some risk metrics are included in the model as additional criteria to be optimized. A detailed description of these metrics is presented next.

(i) Downside Risk (DR): $D R$ represents the positive deviation from a defined target (generally denoted by $\Omega$ ). $D R$ can be expressed as shown in Eq. (3):

$$
D R_{\Omega}=E\left[\delta_{\Omega \mathrm{s}}\right]=\sum_{s} \operatorname{prob}_{\mathrm{s}} \delta_{\Omega \mathrm{s}}
$$

where

$$
\delta_{\Omega \mathrm{S}}=\left\{\begin{array}{cc}
\Omega-\text { Profit }_{s} & \text { if } \text { Profit }_{s}<\Omega \\
0 & \text { otherwise }
\end{array}\right\} \quad \forall s \in \mathrm{S}
$$

Here, Profit $t_{s}$ accounts for the profit in scenario $s \in S$.

(ii) Risk: This metric also requires the definition of a target, but it measures the probability of not achieving this target rather than the deviation from it. Risk is mathematically expressed as follows:

$$
\operatorname{Risk}_{\Omega}=\sum_{s} \operatorname{prob}_{s} Z_{\Omega s}
$$




$$
Z_{\Omega \mathrm{s}}=\left\{\begin{array}{llr}
1 & \text { if } & \text { Profit }_{s} \leq \Omega \\
0 & & \text { otherwise }
\end{array}\right\} \quad \forall s \in \mathrm{S}
$$

Notice that even if both, $D R$ and Risk, provide a measure of the deviation of the solution from a given target, the calculation of the latter involves a bigger computational effort since it requires the definition of binary variables for each scenario.

(iii) Value at Risk (VaR) and Opportunity Value (OV): These metrics assess the performance of a solution in a given region of the cumulative probability curve. More precisely, the $V a R$ is the difference between the expected profit and the profit for a cumulative probability of 5\%, while the $O V$ is conceptually equal to $V a R$, but covers the upper side of the cumulative risk curve (typically a percentile of $95 \%$ ). Hence, those values are usually used together in order to explore both sides of the cumulative risk plot.

(iiii) Worst Case (WC): The WC has been adopted as an alternative to control the probability of meeting unfavorable scenarios. It leads to a simple formulation that requires a low computational effort (see Eq.(7)).

$$
W C \leq \text { Profit }_{s} \quad \forall s \in \mathrm{S}
$$

For more details about the above risk metrics and their implementation in supply chain models, the reader is referred to the works by Aseeri et al. (2004), Aseeri and Bagajewicz (2004), Bonfill et al. (2004), Barbaro and Bagajewicz (2004) and Applequist et al. (2000). Finally, the stochastic model that optimizes a set of risk metrics can be formally expressed as follows:

$$
\begin{array}{ccl}
\text { (P) } \max _{x y} & \left\{f_{1}\left(x, y_{s}, \theta\right), \ldots, f_{o b}\left(x, y_{S}, \theta\right), \ldots, f_{|O B|}\left(x, y_{s}, \theta\right)\right\} \\
\text { s.t. } & h\left(x, y_{s}, \theta\right)=0 & \forall s \in \mathrm{S} \\
g\left(x, y_{s}, \theta\right) \leq 0 & \forall s \in \mathrm{S} \\
& x \in \mathrm{X}, y_{S} \in \mathrm{Y}, \theta \in \Theta &
\end{array}
$$

Where $f_{o b}$ represents the different objective functions of the problem (e.g. $f_{1}=$ expected profit, $f_{2}=-D R, f_{3}=-R i s k$, etc.). A detailed description of the expected profit calculation is presented in Appendix A. Note that our approach is general enough to accommodate other risk metrics as well.

\subsection{Solution strategy (Sample Average Approximation algorithm).}

Solving P (step 2 in Fig. 2) is challenging due to the number of scenarios and objectives. To expedite its solution, we propose a strategy based on the Sample Average Approximation (SAA) algorithm. The SAA has its roots in the so called stochastic counterpart (Rubistein and Shapiro, 1990) and the sample path optimization methods (Plambeck et al., 1996). 
We make use of the following decomposition strategy to solve Model P (Shabbir and Shapiro, 2002; Kostin et al., 2012). We first solve the model in its deterministic form considering only one scenario at a time and optimizing the profit as the unique objective. Then, we fix the values obtained for the first-stage variables (i.e., the design of the supply chain) and optimize again the expected profit in Model $\mathrm{P}$, but this time considering all the $|S|$ scenarios. This process is repeated recursively by replacing the parameters values used in the deterministic model solved in step one (corresponding to one particular scenario) by those associated with another scenario in order to obtain the optimal supply chain design for each of the remaining $|S|-1$ scenarios so that, at the end, $|S|$ different solutions are generated. Note that the standard SAA approximates the solution of a single-objective stochastic problem by solving a series of stochastic subproblems, each of them with fewer scenarios than the original full space stochastic model (Verweij et al., 2002; Santoso et al., 2005). These scenarios, sampled from the original set of scenarios, approximate the expected objective value of the original problem. After solving each subproblem, the first stage decisions are fixed in the original model, which is solved iteratively for all the solutions generated in the subproblems. The solution among the ones generated with the subproblems that performs best in the full space model is finally used to approximate the global optimum of the original stochastic model. Hence, in our case, the subproblems contain one single scenario (i.e., they are deterministic), as opposed to what happens in the standard SAA, which solves subproblems with more than one scenario.

The overall algorithm is as follows.

1. Define the set of scenarios $S$ and initialize the raw set of solutions $R S S=\varnothing$

2. For $e=1: 1:|S|$

2.1. Solve Model $\mathrm{P}$ considering only the scenario with index $e$ (say scenario $\mathrm{s}_{\mathrm{e}}$ ). Let solution $\bar{x}^{*}$ be the value of the first stage variables in this problem.

2.2. Fix first stage variables as in $\bar{x}^{*}$.

2.3. Solve Model $\mathrm{P}$ including all the $|S|$ scenarios. Let $\bar{x}^{*}, \bar{y}_{s}^{*}$ be the values of the first and second stage variables in the full optimal solution to this problem (the solution with optimal second-stage variables for the first-stage values generated in step 2.1).

2.4. Calculate risk metrics.

2.5. Make $R S S=R S S \cup \bar{x}^{*}$ and free the first-stage variables,

3. End for.

Note that even if Model $\mathrm{P}$ is a multi-criteria model, the only objective function considered during the algorithm is the profit maximization (i.e., risk metrics are calculated in parallel during the process, but they never act as objective functions). The reason for this is two-fold. First, it is not possible to optimize any risk metric in step 2.1 , as the model solved in this step is essentially deterministic (i.e., it considers one scenario only). Second, the model considered in step 2.3 is 
indeed stochastic and could therefore allocate any risk metric as objective function, yet this would entail no significant benefit since the risk can be mainly controlled through modifications in the design of the supply chain, which has already been fixed in a previous step (step 2.2).

It is important to highlight that the problem with first and second-stage variables is not rigorously solved and, consequently, the proposed methodology cannot guarantee global optimality for the solutions obtained. However, the proposed approach is indeed an approximation method (i.e., heuristic) to solve the full space multi-objective stochastic model. Recall that for every set of first stage designs calculated in step 2.2, we determine the corresponding second-stage variables in step 2.3 following the general scheme of the SAA and considering the entire uncertain parameters space. This part of the algorithm (step 2.3) assesses whether the designs are feasible in all the scenarios. Consequently, even if the solutions obtained with the proposed method may be dominated by others which are overlooked, they are proved to be feasible and therefore eligible to be ranked by the Pareto filters as long as they successfully pass step 2.3 of the algorithm. Otherwise (i.e., if they render unfeasible), they are discarded.

\subsection{Normalization of solutions.}

The SAA method provides as outcome a raw set of solutions (RSS) to problem P. A normalization step is applied to facilitate the post-optimal analysis of these solutions. Different normalization algorithms can be applied at this point (see Bolstad et al. (2003)). In this work, we use the basic interpolation method, which is formulated as follows:

$$
\hat{f}=\hat{f}_{l o}+\left(\hat{f}_{u p}-\hat{f}_{l o}\right) \frac{f-f_{l o}}{f_{u p}-f_{l o}}
$$

Here, $\hat{f}$ represents the normalized value (which varies between bounds $\hat{f}_{l o}=0$ and $\hat{f}_{u p}=1$ ) associated to the real value $f$, while $f_{l o}$ and $f_{u p}$ represent respectively the minimum and maximum values taken by this objective among the raw set of solutions RSS. At the end of this step, a normalized set of solutions NSS is obtained.

\subsection{Application of Pareto filters.}

Model P potentially contain an infinite number of solutions from which decision-makers should identify the ones that better reflect their preferences. To facilitate this task, two Pareto filters are applied to narrow down the number of Pareto solutions and retain for further inspection solutions showing better overall performance (discarding in turn the rest). Two filters are applied. The first one (Smart filter) eliminates repeated and suboptimal (dominated) solutions from the set as described in Mattson et al. (2004). The second filter (Order of efficiency filter) ranks the Pareto points according to the concept of efficiency of order $k$, as described in Das et al. (1999). To this end, the order of efficiency filter is applied recursively for descending orders of efficiency until no solutions are found. Further details about these Pareto filters can be found elsewhere (Antipova et al., 2015; Pozo et al., 2012a). The Pareto filter algorithm is described next and illustrated in Fig. 3. 
1 We first define the objectives to be analyzed $o b \in O B$ and set a tolerance value for the Smart Filter

$2(\Delta t)$. Let $N O O$ be the number of objectives considered.

3 1. Apply Smart filter to solutions NSS considering objectives $o b \mid o b \in O B$ using tolerance $\Delta t$. $4 \quad$ Let $\mathrm{M}^{\prime}$ be the set of solutions retained after the application of the filter.

52 . If $M^{\prime}=\varnothing$, stop, further reduction is not possible. Else:

2.1. For $k=N O O: 1: 1$

2.1.1. Apply Order of efficiency filter to solutions $M^{\prime}$ for $k$. Let $V_{k}$ be the set of solutions which are efficient of order $k$.

$$
\text { 2.1.2. Make } M^{\prime}=V_{k} \text {. }
$$

\subsection{End for}

3. End if.

As seen, the algorithm starts by applying the smart filter for a given tolerance. Then, the order of efficiency filter is applied until further reductions in the Pareto set cannot be attained.

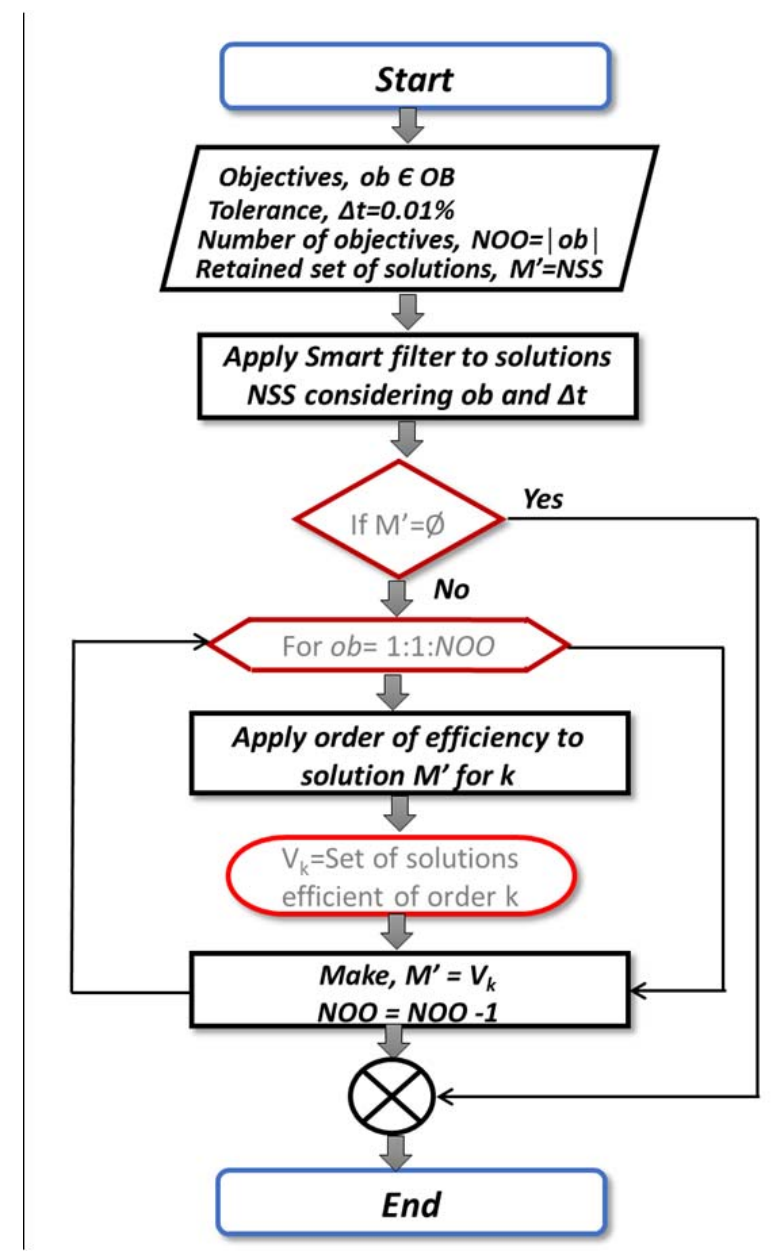

Fig. 3. Detailed description of the Pareto filter procedure used to reduce the set of optimal solutions.

A detailed description of each step in the above algorithm follows. 


\subsubsection{Smart Pareto filter.}

3 This filter uses a defined tolerance value $(\Delta t)$ to discard solutions that are potentially repeated or 4 redundant. The method selects one solution and scans the tolerance area in order to find and discard 5 points falling within it, thereby removing dominated solutions considering such a tolerance. The 6 tolerance value is defined by the user and has a strong impact on the outcome of the algorithm. If it 7 is too large, the final set of alternatives will be very small, but appealing solutions may be lost, 8 whereas if it is too small the opposite situation will occur.

9 Fig.4 illustrates the idea behind the Smart Pareto filter. Given the set of solutions $\mathrm{Sol}_{\mathrm{s}}, \mathrm{Sol}_{1}$ is taken as core solution and compared with the rest. The dominated solutions and those inside the tolerance area (shaded region) are removed from the pool. Afterwards, the nearest Pareto solution will be selected as core and the operation will be performed again until no Pareto solutions remain unexplored. In this example, solutions $\mathrm{Sol}_{7}, \mathrm{Sol}_{8}$ and $\mathrm{Sol}_{9}$ are dominated solutions and are removed from the pool of solutions when solutions $\mathrm{Sol}_{2}$ and $\mathrm{Sol}_{3}$ are evaluated, respectively. Additionally, even if solution $\mathrm{Sol}_{10}$ is Pareto optimal, it lies in the tolerance area of solution $\mathrm{Sol}_{3}$, so it is considered indistinguishable from it and thus eliminated.

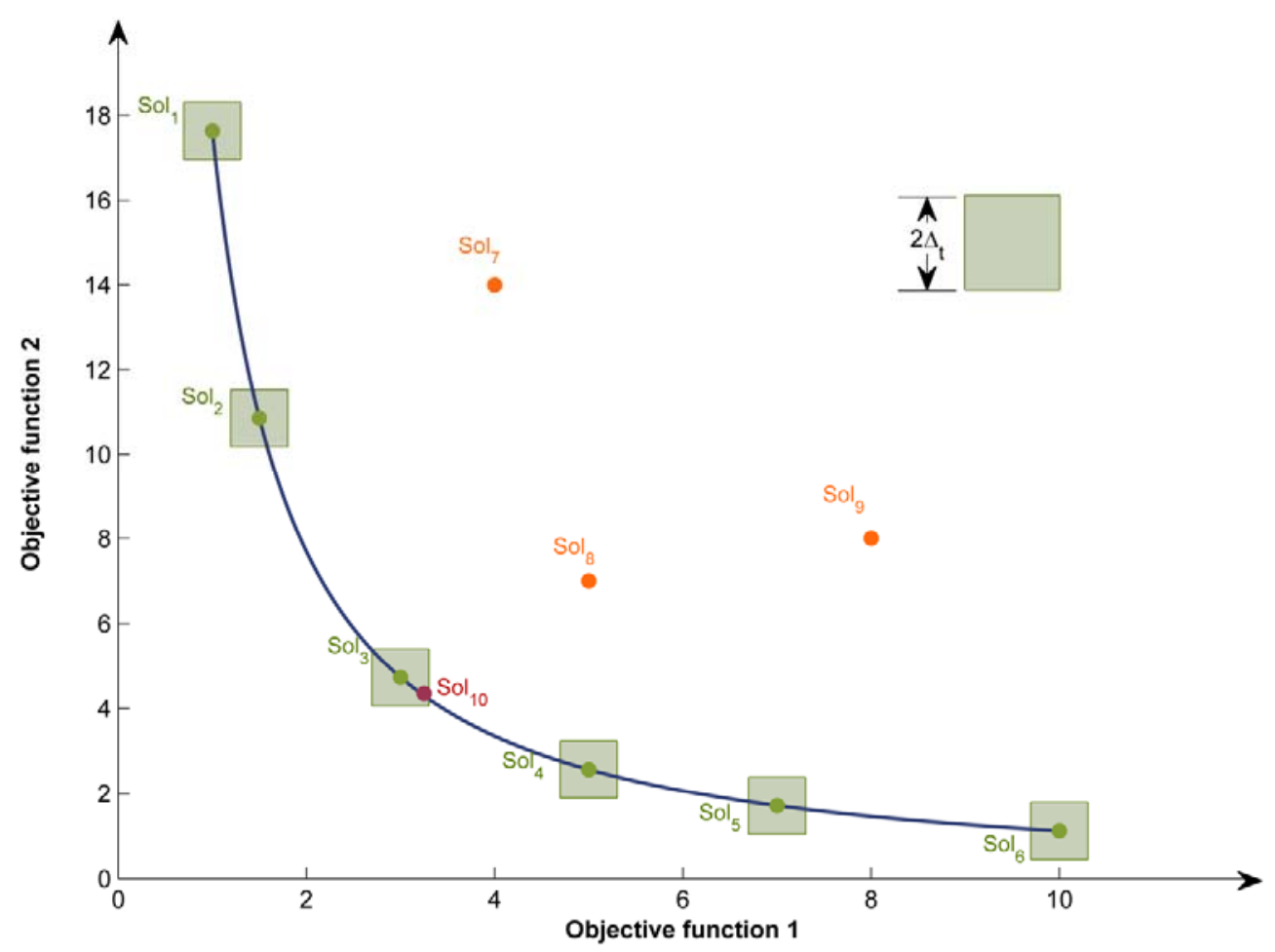


Fig 4. Representation of the Smart Pareto filter algorithm. A solution is considered indistinguishable from other one if the first (red point) falls into the tolerance area (shaded gray zone) of the solution under analysis (green points). Dominated solutions (orange points) are also identified and eliminated.

A more formal mathematical description of the algorithm is presented next. Let $\mathrm{SS}_{\mathrm{c}}$ be one solution of the normalized set of solutions (NSS) obtained through steps 2 to 4 of the proposed methodology approach, and $o b$ one of the objectives considered. The filter comprises the following steps.

1. Initialize a set of rejected solutions $(\mathrm{RS}=\varnothing)$, a set of candidate solutions $\left(\mathrm{M}^{\prime}=\emptyset\right)$ and 2 counters ( $c^{\prime}$ and $\left.c c^{\prime}\right)$ which are initially set to zero. Additionally define a tolerance value $(\Delta t)$.

2. While $\mathrm{c}^{\prime}<|N S S|$

$$
\text { 2.1. } c^{\prime}=c^{\prime}+1
$$

2.2. If $\nexists S S_{C^{\prime}} \mid S S_{C^{\prime}} \in N S S$, return to 2.1. Else:

2.3. While $\mathrm{cc}^{\prime}<|N S S|$

2.3.1. $\mathrm{cc}^{\prime}=\mathrm{cc}^{\prime}+1$

2.3.2. If $\nexists S_{C C^{\prime}} \mid S_{C C^{\prime}} \in N S S$, return to 2.3.1. Else:

2.3.3. If $\mathrm{c}^{\prime}=\mathrm{cc}^{\prime}$, return to 2.3.1. Else, if $\mathrm{SS}_{c^{\prime}, \mathrm{ob}}-S S_{c c^{\prime}, \mathrm{ob}}<\Delta \mathrm{t} \forall o b$, let $R S=R S \cup S S_{c c^{\prime}}$ and $N S S=N S S \backslash S S_{C C l}$.

2.4. End while

2.5. Restart counter $\mathrm{cc}^{\prime}=0$

3. End while

4. Make $\mathrm{M}^{\prime}=$ NSS

\subsubsection{Order of efficiency filter.}

This filter makes use of the concept of order of efficiency, which assesses the "level of optimality" of a solution. The order of efficiency was originally introduced by Das (1999), and has been recently applied to metabolic engineering (Pozo et al., 2012b) and desalination plants (Antipova et al., 2015).

A solution is said to be efficient of order $k$ if it is not dominated by any other solution in any of the possible $k$-elements subsets of objectives. In the above definition, $k$ represents the size of the subsets, that is, the number of objectives which form the subset (i.e. $1 \leq k \leq \mathrm{NO}$, being NO the number of objectives). Using the same nomenclature, statements and variable definitions from the previous section, a mathematical representation of the order of efficiency concept is presented next.

A solution $x x^{*} \epsilon M^{\prime}$ is efficient of order $k$, if it is not dominated by any other solution $x x^{* *} \epsilon M^{\prime}$ in any of the possible subsets of $k$ objectives. In other words, given any of the sets of $k$ objectives, it does not exist any feasible solution $x x^{* *}$ such that $f_{o b}\left(x x^{* *}\right) \leq f_{o b}\left(x x^{*}\right)$ for all ob $\in$ $\left(o b_{1}, o b_{2} \ldots o b_{k}\right)$ and at the same time $f_{o b}\left(x x^{* *}\right)<f_{o b}\left(x x^{*}\right)$ for at least one $o b \in\left(o b_{1}, o b_{2} \ldots o b_{k}\right)$. From this definition, it follows that if $x x^{*}$ is efficient of order $k$, it is also efficient of any order greater than $k$. Note that lower orders of efficiency reflect a better balance among objectives in the solution and, in some way, the more appealing for decision-makers. 
The concept of Pareto efficiency of order $k$ is illustrated in Fig. 5, which shows the parallel coordinates plot where each line represents one of the 4 solutions retained in the previous example (i.e., $\mathrm{Sol}_{1}, \mathrm{Sol}_{2}, \mathrm{Sol}_{3}$ and $\mathrm{Sol}_{4}$ ). As can be seen, solutions $\mathrm{Sol}_{1}, \mathrm{Sol}_{2}$ and $\mathrm{Sol}_{4}$ are Pareto optimal, that is, they are at least efficient of order 3 (recall that due to the normalization step, values equal to 0 is the best objectives performance and vice versa ). On the contrary, $\mathrm{Sol}_{3}$ is an inefficient solution because it is dominated by Sol . The next step is to check whether solutions $\mathrm{Sol}_{1}, \mathrm{Sol}_{2}$ and $\mathrm{Sol}_{4}$ are also efficient of lower order, for which all the possible subsets of $k<3$ objectives must be considered. For instance, solutions $\mathrm{Sol}_{2}$, and $\mathrm{Sol}_{4}$ are dominated by $\mathrm{Sol}_{1}$ in subset $\left\{o b_{2}, o b_{3}\right\}$, so they are no longer candidates to be efficient of order 2. Conversely, $\mathrm{Sol}_{1}$ is not dominated neither in subset $\left\{o b_{1}, o b_{3}\right\}$ nor in $\left\{o b_{1}, o b_{2}\right\}$ and is therefore efficient of order 2. An inspection of subsets of one objective reveals that $\mathrm{Sol}_{1}$ is dominated by both $\mathrm{Sol}_{2}$ and $\mathrm{Sol}_{4}$ in $\left\{o b_{1}\right\}$, and thus it is not efficient of order 1. As a result, the minimum order of efficiency for $\mathrm{Sol}_{1}, \mathrm{Sol}_{2}$ and $\mathrm{Sol}_{4}$ is 2, 3, 3, while $\mathrm{Sol}_{3}$ is inefficient. This approach allows ranking the Pareto solutions according to their efficiency order. Hence, solution $\mathrm{S}_{1}$ would be the most appealing, since it shows better average performance when considering all of the objectives simultaneously.

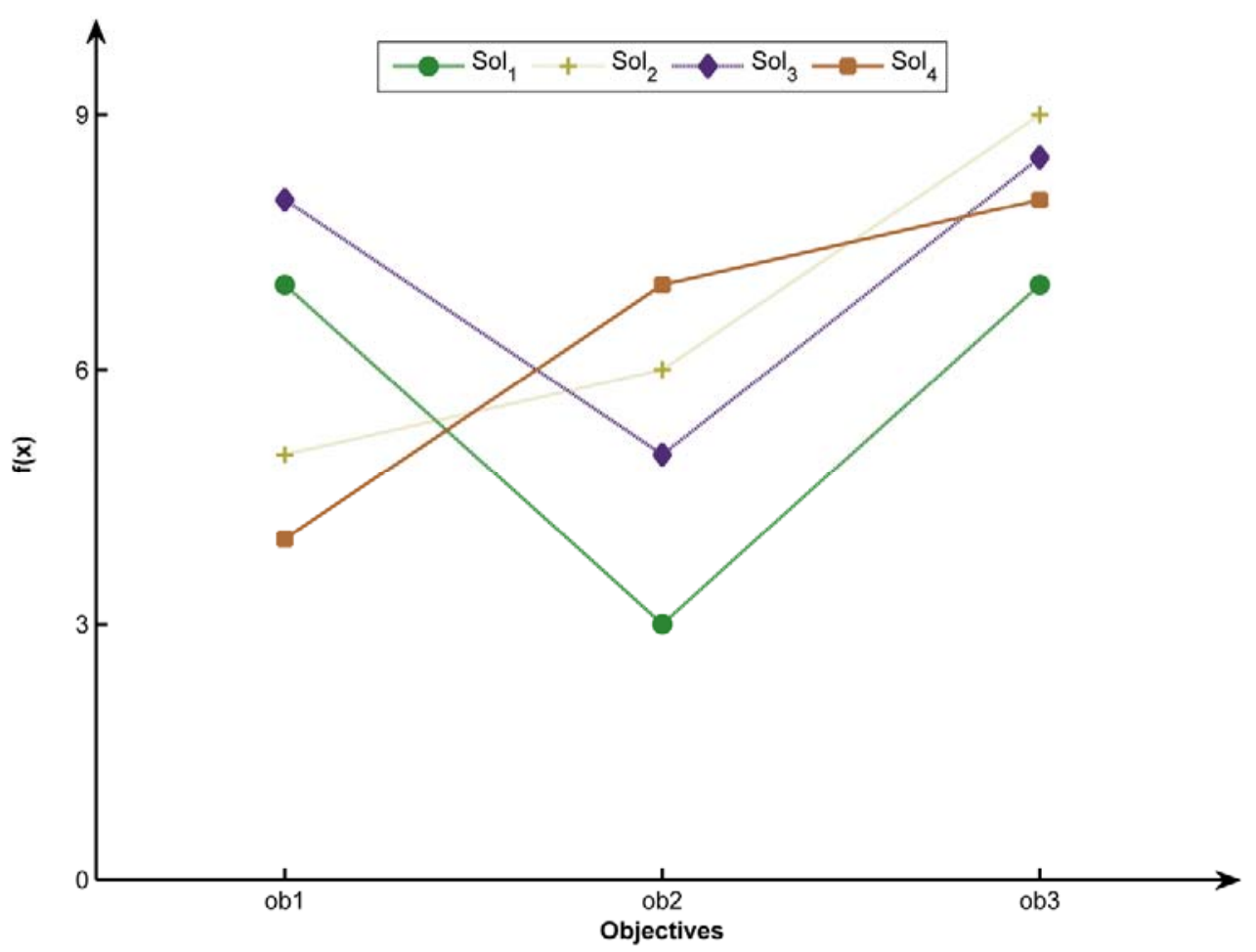

Fig. 5. Illustration of the order of efficiency filter. There are 4 solutions that have to be minimized for all the 3 objectives considered. $\mathrm{Sol}_{1}$ is efficient of order 2 whereas $\mathrm{Sol}_{2}$ and $\mathrm{Sol}_{4}$ are efficient of order 3 , and $\mathrm{Sol}_{3}$ is inefficient (i.e., not Pareto optimal).

Note that Pareto filters imply stronger conditions than the conventional Pareto optimality criterion. This concept avoids the use of any arbitrary "criterion of merit" or visualization technique, thereby making the approach suitable for high-dimensionality problems (Pozo et al., 2012b; Das, 1999). 


\section{Case study: Multi-criteria Design and Planning of SC under uncertainty}

The proposed approach is now illustrated through its application to the design and planning problem of a supply chain with embedded batch facilities. The system considers 3 raw material sources, which can feed 5 potential batch plants with up to 3 phases. Different discrete sizes are considered for each batch unit $\left(0.3 \mathrm{~m}^{3}, 0.5 \mathrm{~m}^{3}, 0.75 \mathrm{~m}^{3}, 1 \mathrm{~m}^{3}\right.$ and $\left.1.2 \mathrm{~m}^{3}\right)$ and intermediate storage tank $\left(3 \mathrm{~m}^{3}, 5\right.$ $\mathrm{m}^{3}, 10 \mathrm{~m}^{3}$ ). Final products can be stored in 3 warehouses before being sent to 3 customer zones. The resulting multi-scenario MILP problem is described in detail in Appendix A, while the remaining system parameters are provided in Appendix B. The model optimizes the design of the required supply chain network (i.e. allocation decisions, production and capacity levels and flows between the SC nodes) considering the effects of the potential planning decisions. The model also determines the optimal design of the embedded batch plants (i.e. structure of the plants) considering parallel unit duplication, allocation of storage tanks, and unit size. Binary variables are used in the mathematical model in order to represent the allocation decisions of a particular site/unit.

Product demand was considered as the only uncertain parameter and modeled through a normal distribution. 100 scenarios were generated via Monte Carlo sampling in order to discretize the normal distributions, assuming the mean values in Table B.2 (See Appendix B) and a variance of $15 \%$. It is important to highlight that Monte Carlo sampling is less efficient than other sampling techniques, such as Sobol sampling, polynomial-based methods (cubature formula) (Bernardo et al., 1999) and methods based on low-discrepancy samples (also known as quasi-Monte Carlo methods)(Diwekar and Kalagnanam, 1997). However, here it is used as a crude method to illustrate the generation of scenarios in the proposed methodology. The minimum number of scenarios was determined by two methods. First, by solving the SAA for an increasing number of scenarios and then stopping when the difference between the expected profit of the best two consecutive solutions provided by the SAA (i.e., the difference between the best expected profit solution provided by the SAA for a given number of scenarios, and the best expected profit solution for the same number of scenarios plus one) was less than $5 \%$. Second, we applied the methodology proposed by Law and Kelton (2000), which was recently applied to stochastic problems (Sabio et al., 2014). This approach was solved considering a relative error of 0.1 and a confidence level of $1 \%$ (see . Appendix C). For these settings, the minimum number of scenarios was 73 .

The deterministic model contains 3,222 equations, 2,086 continuous variables and 223 binary variables, while the stochastic one with 100 scenarios has 178,552 equations, 153,061 continuous variables and the same number of binary variables (223). All the runs were implemented in GAMS 23.9 and solved using CPLEX on a Windows XP computer with Intel ${ }^{\circledR C} \mathrm{Core}^{\mathrm{TM}}{ }^{\mathrm{i}} 7 \mathrm{CPU}(920)$ $2.67 \mathrm{GHz}$ processor with $4.00 \mathrm{~GB}$ of RAM. It takes approximately 27.3 seconds to generate each solution of the deterministic model. It is important to mention that the stochastic model that includes all the scenarios and maximizes the expected profit as unique criterion cannot be solved in 86,400 seconds (24 hours) (i.e., after this CPU time, CPLEX is unable to close the optimality gap below $5 \%$ even when optimizing only the expected profit; so much larger CPU times are expected when dealing with several risk metrics simultaneously).

As shown in Table 1, two cases differing in the risk metrics are investigated. The targets required in the calculations of the risk metrics were defined as follows. We first applied the SAA and plotted 
the cumulative curve (Fig. 6) for each deterministic design generated for each scenario. We then defined the target values by identifying the lower, middle and upper parts of these cumulative distributions. Each curve in Fig. 6 represents a specific SC configuration with associated planning decisions. Expected profit values range from $\$ 530,000$ to $\$ 1,334,000$. In the figure, we have highlighted the solution with maximum expected profit (maxEProfit) as well as two curves that may be appealing for risk-averse and risk-taker decision makers. A Risk-Averse solution corresponds to that in which lower probabilities of small/high profits are found. On the contrary, a solution with larger probabilities of high profits (at the expense of increasing as well the probability of low benefits) is appealing for a Risk-Averse behavior.

Solutions behave differently in the uncertain parameters space, as can be noticed by the performance of the three highlighted solutions. For instance, maxEProfit has a probability of $19 \%$ of not exceeding a target value of $\Omega=\$ 1.00 \mathrm{M}$, while this probability increases gradually to $25 \%$ and $55 \%$ in the Risk-Averse and Risk-Taker solutions, respectively. Here, the maxEProfit solution represents a very conservative choice that behaves better than the remaining solutions for a wide range of target values $(\Omega<\$ 1.15 \mathrm{M})$, however for higher target values this solution shows poor performance. Notice that the better performance attained in the Risk-Averse and Risk-Taker solutions in the upper part of the probability curve is obtained at the expense of a drop in their expected profit. For instance, the Risk-Taker and Risk-Averse solutions show expected profits of $\$ 971,179$ and $\$ 1,057,684$, respectively, whereas the maximum expected profit is $\$ 1,100,211$. Between the Risk-Taker and Risk-Averse solutions, there are many intermediate solutions behaving in different ways.

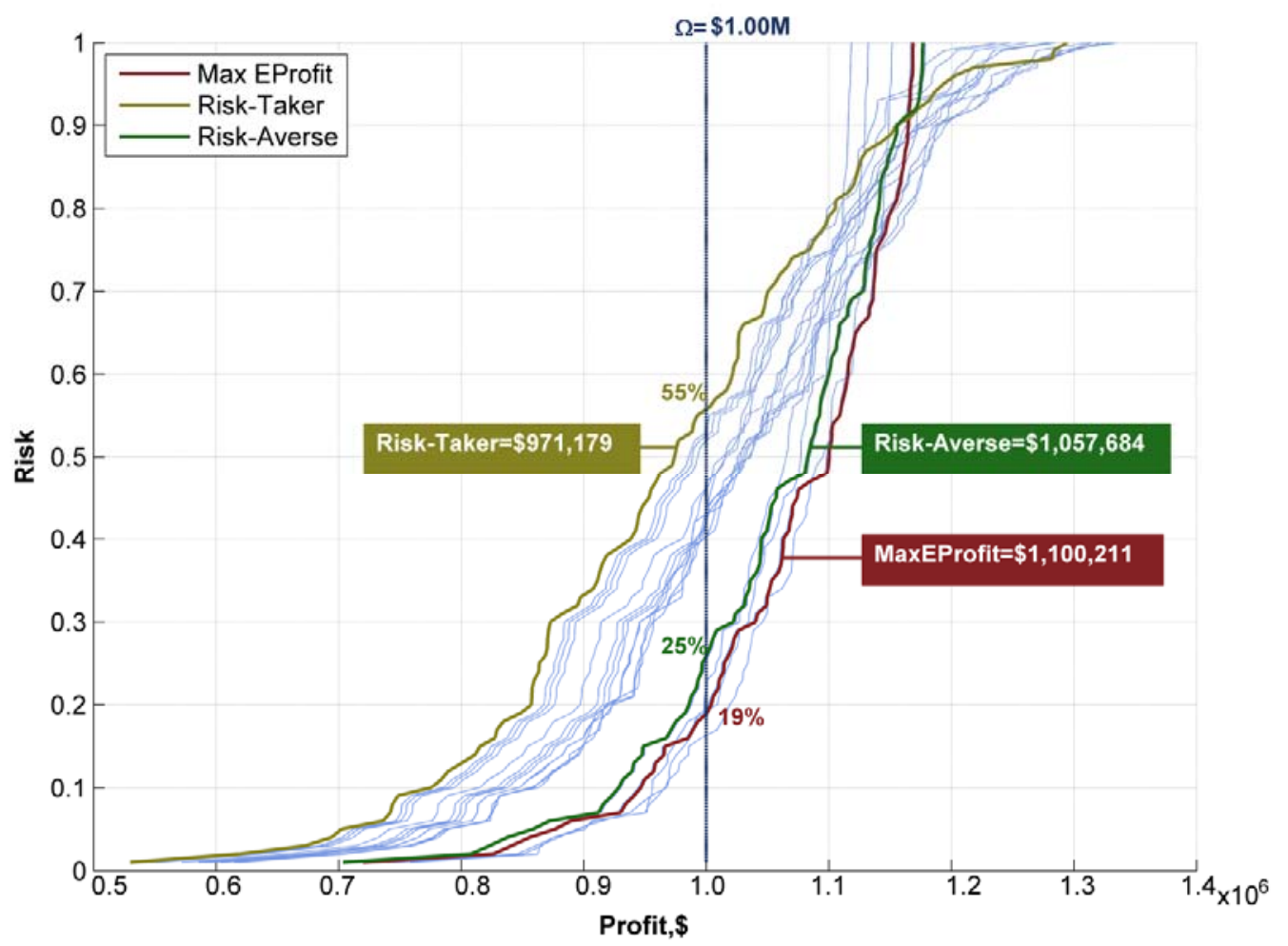


2 All the solutions show essentially the same overall supply chain configuration (see Fig. 7), but 3 differ in the detailed design of the plants, which are explained later in the article. More precisely, 4 they all select plant L4 regardless of the uncertain parameters values, mainly because the required 5 investment and production costs are the lowest. Raw material site S2 supplies all the materials 6 required for producing the four products, because the distribution costs between S2 and L4 are 7 cheaper. The products are delivered to two warehouses, M1 and M3.

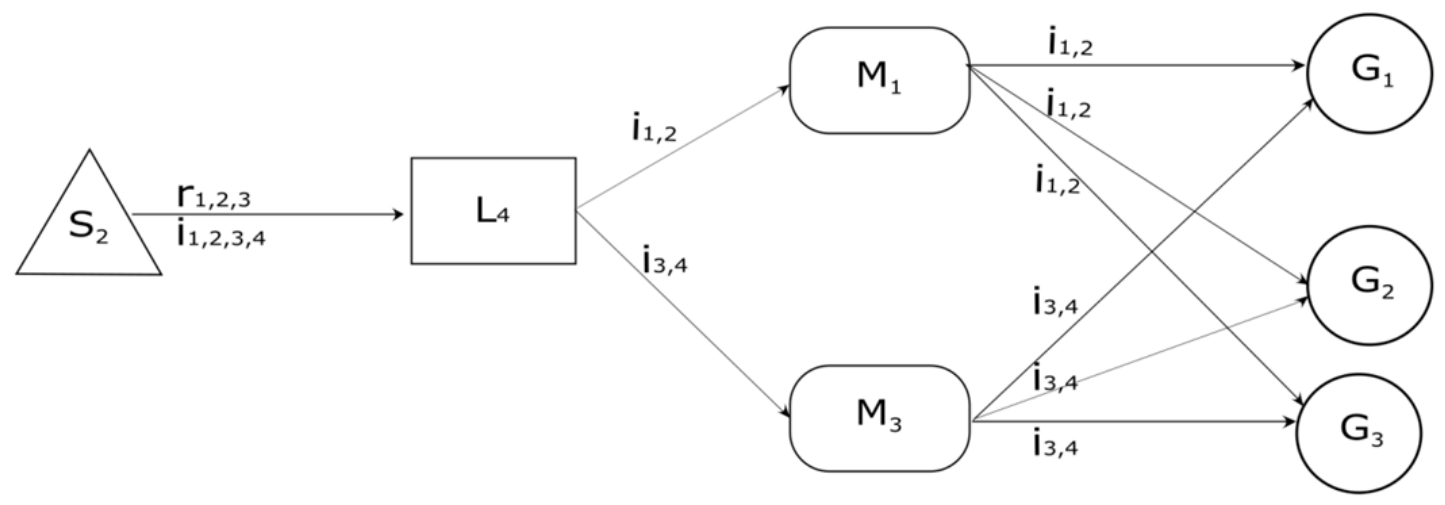

Fig.7 optimal SC design for the 100 demand scenarios.

Table 1. List of objectives and target values considered in this work for both cases. Target values $\Omega$ are expressed in $€ \cdot 10^{3}$.

\begin{tabular}{cc|cc}
\hline \multicolumn{2}{c|}{ First Case } & \multicolumn{2}{c}{ Second Case } \\
\hline Objective/metric & Target value & Objective/metric & Target value \\
\hline Eprofit & N/A & Eprofit & $N / A$ \\
Worst Case (WC) & N/A & & $\Omega=530$ \\
Downside Risk (DR) & $\Omega=800$ & & $\Omega=584$ \\
& $\Omega=950$ & & $\Omega=637$ \\
Value at Risk (VaR)* & $\Omega=1,050$ & & $\Omega=691$ \\
Opportunity Value (OV)* & $9 \%$ & & $\Omega=745$ \\
& & & $\Omega=798$ \\
& & Risk & $\Omega=852$ \\
& & $\Omega=906$ \\
& & $\Omega=959$ \\
& & $\Omega=1,013$ \\
& & $\Omega=1,066$ \\
& & $\Omega=1,120$ \\
& & & $\Omega=1,174$ \\
& & & $\Omega=1,227$
\end{tabular}


${ }^{*}$ The percentage target value for $\operatorname{VaR}$ and $O V$ are the probability value in the cumulative plot.

\subsection{First case: Expected profit, worst case, downside risk, value at risk and opportunity value} Here, we consider $W C, D R, V a R$ and $O V$ as performance criteria (objectives) in addition to Eprofit. For the $D R$ calculation, 3 target values were used corresponding to the lower, middle and upper parts of the cumulative distribution curve. For the VaR and $O V$, the standard 5\% and $95 \%$ percentiles were set (See Table 1).

After the application of our proposed algorithm, 100 solutions were obtained, each one with specific values of the decision variables, expected cost and financial risk metrics. From here, we produced a $100 \times 7$ matrix (henceforth known as matrix N) using the values of each performance criteria in each scenario. Matrix $\mathrm{N}$ is normalized according to the procedure described in section 3.3. Note that some of the deterministic solutions may be suboptimal (in the space of the objectives considered in the analysis), or repeated (i.e. the model yields the same first-stage decision values when solved for two different scenarios). The Pareto filters were applied next using this matrix.

The Smart filter (first step of Pareto filters, section 3.4) was executed with a tolerance value of $\Delta t=0.01 \%$. As a result, the number of solutions was reduced in $80 \%$ (i.e. from 100 to 20). Fig. 8 is a parallel coordinates plot that represents in the horizontal axis the normalized objectives and in the vertical one the performance attained by every solution in each such objective. The objectives are normalized as described previously ( 0 is the best value and 1 is the worst). As seen, the dominated solutions are identified and removed by the filter, so finally 20 solutions remain in Fig. 8 (depicted by polylines), which intersect each other in at least one point. 


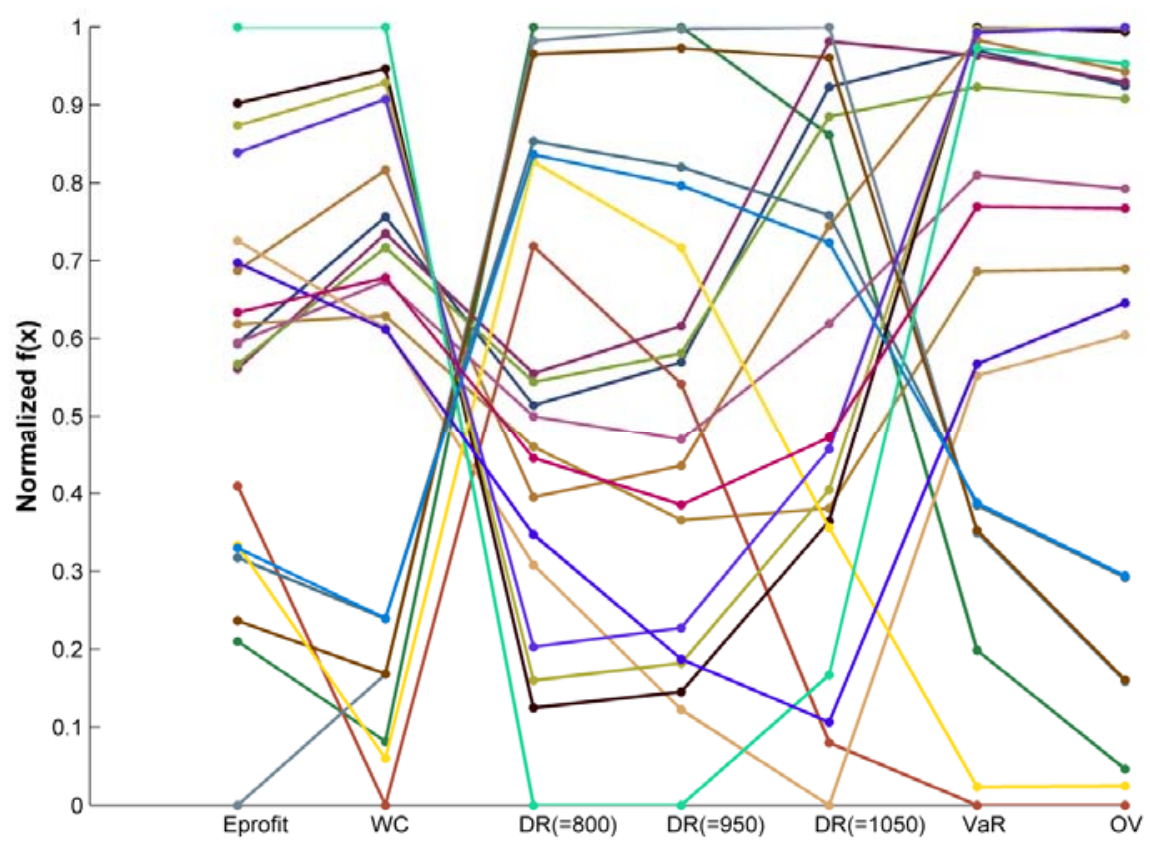

Objectives Fig.8. Parallel coordinate plot showing the interactions and relations among solutions for each objective for
the first case (matrix N).

Remarkably, some objectives behave similarly, that is, when one increases so do the others and vice versa. This is confirmed by the p-values shown in Table 2, which are calculated for the filtered solutions. Two metrics are assumed to be statistically correlated when the p-value is below 0.05 (typical significance value). According to this, metric $\mathrm{DR}(\Omega=1050)$ is uncorrelated with $\mathrm{WC}, \mathrm{VaR}$ and OV (see highlighted values in Table 2).

Table 2. P-value for each pair of objectives considered in this work for filtered solutions in case 1.

\begin{tabular}{lccccccc}
\hline \multicolumn{7}{c}{ P-Value } \\
\hline & Eprofit & WC & DR $(\Omega=800)$ & DR $(\Omega=950)$ & DR $(\Omega=1,050)$ & VaR & OV \\
\hline Eprofit & & & & & & & \\
WC & 0.00 & & & & & & \\
DR $(\Omega=800)$ & 0.00 & 0.00 & & & & & \\
DR $(\Omega=950)$ & 0.00 & 0.00 & 0.00 & & & & \\
DR $(\Omega=1,050)$ & 0.01 & $\mathbf{0 . 4 3}$ & 0.01 & 0.00 & & & \\
VaR & 0.00 & 0.00 & 0.00 & 0.01 & $\mathbf{0 . 7 6}$ & & \\
OV & 0.00 & 0.00 & 0.00 & 0.00 & $\mathbf{0 . 7 4}$ & 0.00 & \\
\hline
\end{tabular}

The order of efficiency step was next applied (second step of Pareto Filters, section 3.4) in order to identify non-dominated solutions in all the subsets of objectives of cardinality $k$. Starting from $k=$ 7 , we reduced gradually the value of $\mathrm{k}$ until no solution satisfied the corresponding optimality level (no solution was optimal for all the subsets of $k$-objectives). For each value of $\mathrm{k}<7$, a reduced 


\begin{tabular}{llllll}
\hline \multicolumn{5}{c}{ Matrix $N$} \\
\hline Order of efficiency & $\mathrm{k}=7$ & $\mathrm{k}=6$ & $\mathrm{k}=5$ & $\mathrm{k}=4$ & $\mathrm{k}=3$ \\
Number of solutions & 20 & 4 & 2 & 1 & 0 \\
\hline
\end{tabular}

subset of solutions was obtained. Table 3 displays the size of the subsets for each order of efficiency, in which a reduction of 80,90 and $95 \%$ (from 20 to 4,2 and 1, respectively) in the number of solutions were obtained using $k=6, k=5$ and $k=4$, respectively.
6

7 To guarantee the quality of the solutions kept in each subset, we analyzed how they perform in each 8 objective. Fig.9(a) shows the lower bound for the solutions (best performance) retained in each 9 subset of $k$-objectives for the group of objectives in matrix N. Note that the lower bound for $\mathrm{k}=7$ is 100 for all of the objectives, since this represents the original solution space (and consequently 11 includes the best solutions identified by the SAA). The solutions efficient of order $k=6$ show similar bounds as those solutions in the original set $(k=7)$, with just a small deviation in the value of Eprofit (the best Eprofit in the original set is $\$ 1,100,211$, and in the set $\mathrm{k}=6$ is $\$ 1,047,408$ ) Moreover, solutions retained for lower orders of efficiency $(\mathrm{k}<6)$, present worse bounds in multiple objectives. On the other hand, Fig.9 (b) shows the upper bound for the solutions retained in each subset of $k$-objectives. Here, the value of all the objectives in subset $\mathrm{k}=7$ is 1 , since it includes the worst performance solution in the original solution space. In this case, a bigger deviation from the original subset $\mathrm{k}=7$ would be preferred, as this would imply that bad solutions would have been discarded. By analyzing simultaneously Fig. 9 (a) and (b), it can be seen that solutions in the subset $\mathrm{k}=6$ show good performance compared with the original set $(k=7)$. Hence, the filter is stopped at $\mathrm{k}=6$, when 4 solutions are kept. This represents an overall reduction of $96 \%$ in the size of the original set of solutions (from 100 to 4). 
(a) Lower bound

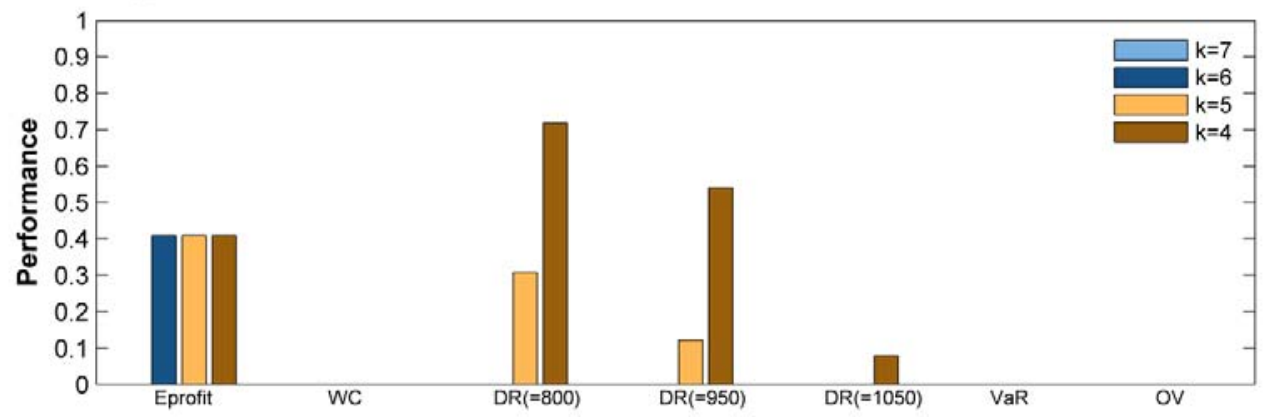

(b) Upper bound

Objectives

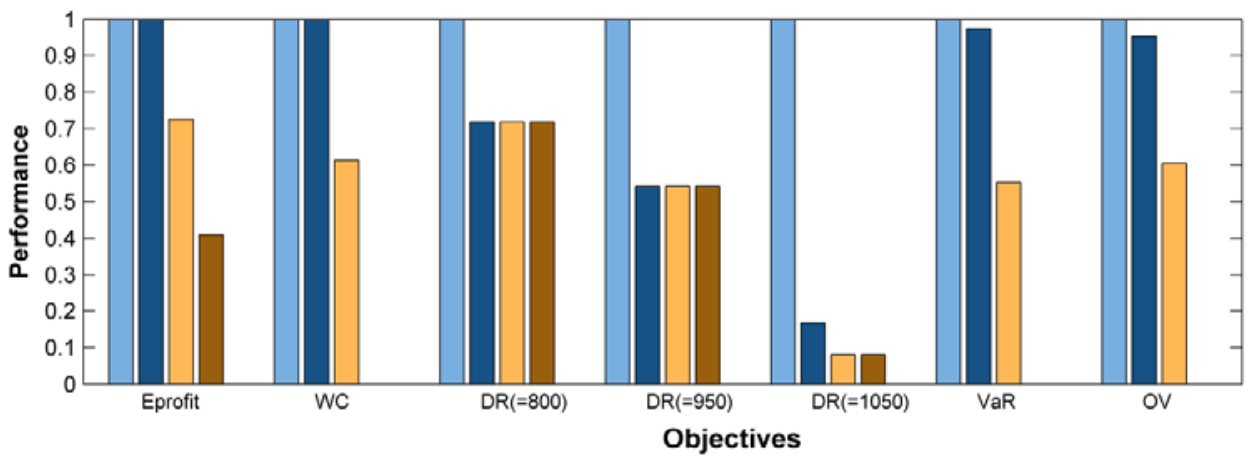

1

4 5

6

8

9

10

11

12

13

(n)

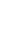
0 1 12

Fig. 9. Normalized bounds for solutions with efficiency of order k for the first case. (a) Lower bound. (b) Upper Bound.

Fig. 10 shows the risk curves associated to each solution for the reduced set of $k=6$, while Fig. 11 and Table 4 show their configurations.

\section{(1)}

Table 4. Batch plant design for the reduced set of solutions in case 1.

\section{First case $(k=7)$}

\begin{tabular}{|c|c|c|c|c|c|c|c|c|c|}
\hline \multirow{2}{*}{ Configuration } & \multirow{2}{*}{$\begin{array}{l}\text { Order of } \\
\text { efficiency }\end{array}$} & \multirow{2}{*}{ Eprofit (M\$) } & \multirow{2}{*}{$\begin{array}{c}* * \text { Demand } \\
\text { Satisfaction }(\%)\end{array}$} & \multicolumn{3}{|c|}{ Batch stage capacities $\left(\mathrm{m}^{3}\right)$} & \multicolumn{3}{|c|}{ *Storage tanks $\left(\mathrm{m}^{3}\right)$} \\
\hline & & & & J1 & $\mathrm{J} 2$ & $\mathrm{~J} 3$ & $\mathrm{~J} 1$ & $\mathrm{~J} 2$ & J3 \\
\hline
\end{tabular}




\begin{tabular}{llllllllll}
\hline 1 & $\mathrm{k}=4$ & 1.047 & 71 & 1 & 0.75 & 0.5 & 0 & 3 & 10 \\
2 & $\mathrm{k}=5$ & 1.007 & 82 & 1.2 & 0.75 & 0.5 & 0 & 5 & 10 \\
3 & $\mathrm{k}=6$ & 0.971 & 100 & 1.2 & 1 & 0.5 & 0 & 0 & 0 \\
4 & $\mathrm{k}=6$ & 1.010 & 82 & 1.2 & 0.75 & 0.5 & 0 & 5 & 0 \\
\hline
\end{tabular}

1

3

*Storage tanks represent the capacity of the tank installed at the exit of each unit J.

** Demand satisfaction level corresponds to the worst case scenario.

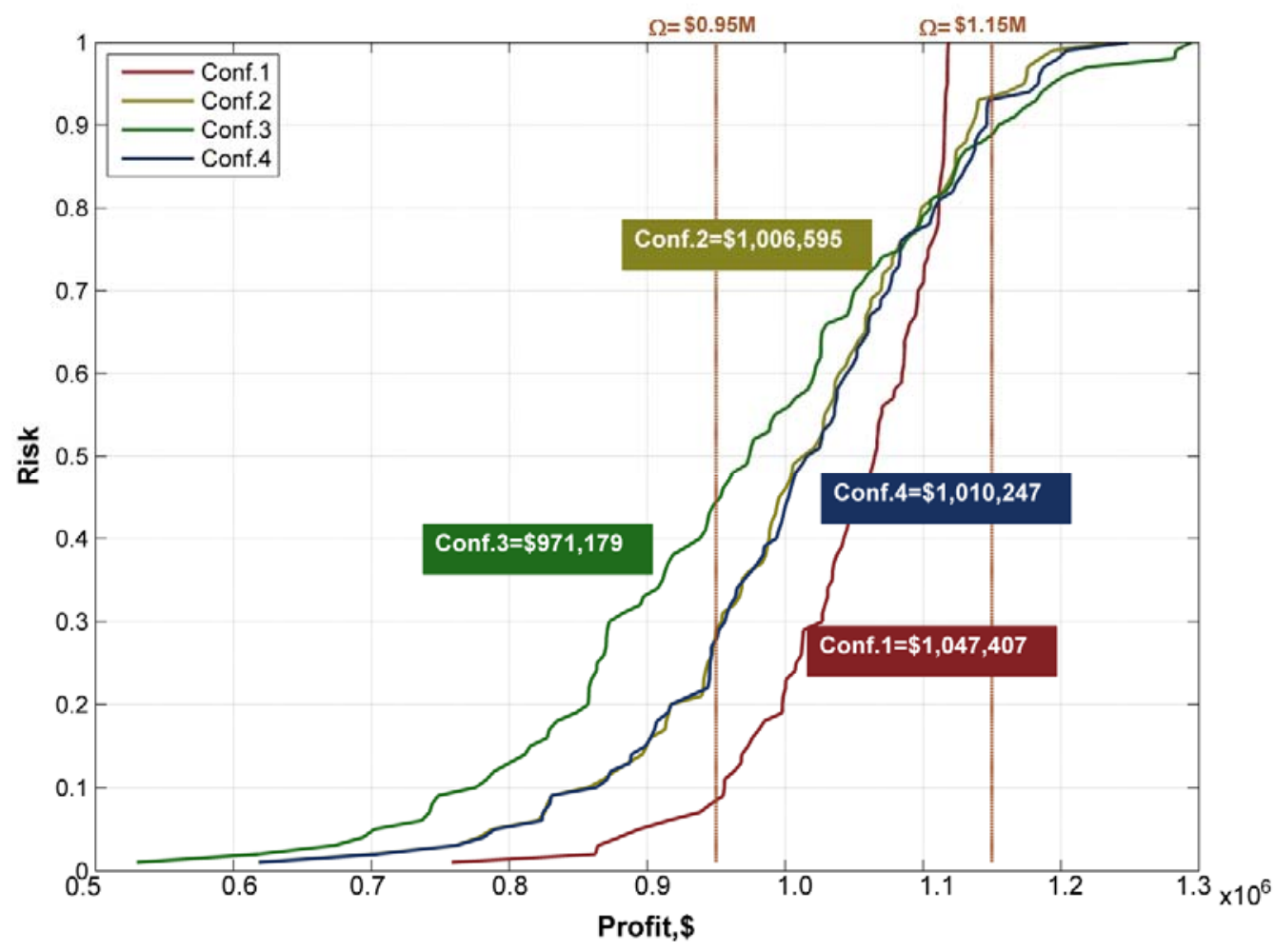

Fig.10. Cumulative risk curves for the solution in the reduced set of case 1.

To get insight into how the model manages the risk associated with the investment, we next study solutions 1 and 4 (configurations 1 and 4, respectively), which are two of the alternatives kept after applying the Pareto filters. Solution 1 reflects a conservative attitude towards risk, with low probabilities of profits below $\$ 0.95 \mathrm{M}(9 \%)$, but a probability of large profits (say above $\$ 1.15 \mathrm{M})$ of $0 \%$. On the other hand, solution 4 reflects a riskier attitude, with a probability of $28 \%$ for profits below $\$ 0.95 \mathrm{M}$, but a larger probability of high profits $(10 \%$ for a target of $\$ 1.15 \mathrm{M})$. As seen in Fig. 11, the risk-averse solution (configuration 1) implements a design with small capacities for the equipment units and storage tanks. This first case study aims to identify a solution reflecting a conservative attitude towards risk, as most of the objectives focus on improving the performance in 
the lower part of the profit distribution. Hence, configuration 1 is therefore kept as it represents a conservative arrangement (smaller equipment sizes and consequently lower potential loses that lead to a higher expected profit).

It is worth to mention that in configuration 1 (see Table 4) demand satisfaction can be compromised and in fact drops to $71 \%$ in the worst case scenario, because the capacity of the supply chain is reduced with the aim of avoiding risk. On the contrary, the risk-taker solution (configuration 4) installs equipment units with higher capacity (and only one storage tank) that can ensure a demand satisfaction of $82 \%$ in the worst case. Finally Solution 3 is the riskiest design, since no single storage is considered and the highest capacities are installed. This leads to higher operation and installation costs as well as less profit on average, but on the other hand allows fully satisfying the demand in all the scenarios. Hence, this design attains higher maximum profits in scenarios with large demands, but this is accomplished at the expense of worse performance in scenarios with low demand.
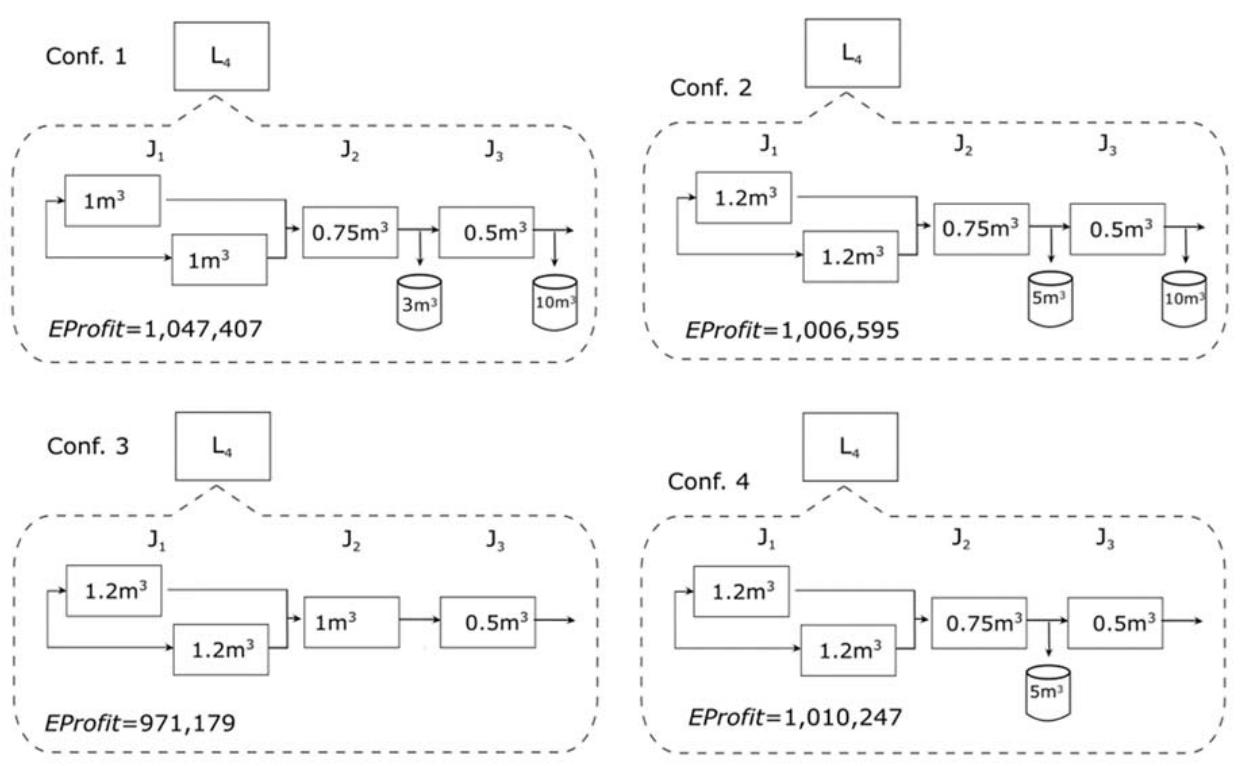

Fig.11. Batch plant configuration scheme for the reduced set of solutions found in the first case study.

\subsection{Second case: Expected profit and risk at different target values}

For this case, we consider Risk as the only additional objective to the expected profit. 16 target values were evenly distributed in the complete solution space for this calculation (see Table 1). 
The first step (Smart filter) was applied considering a tolerance of $\Delta t=0.01 \%$, thereby reducing drastically the number of solutions from 100 to 10 (i.e. a reduction of $90 \%$ ) by removing dominated and repeated solutions. The relationships between objectives are shown in Fig. 12.

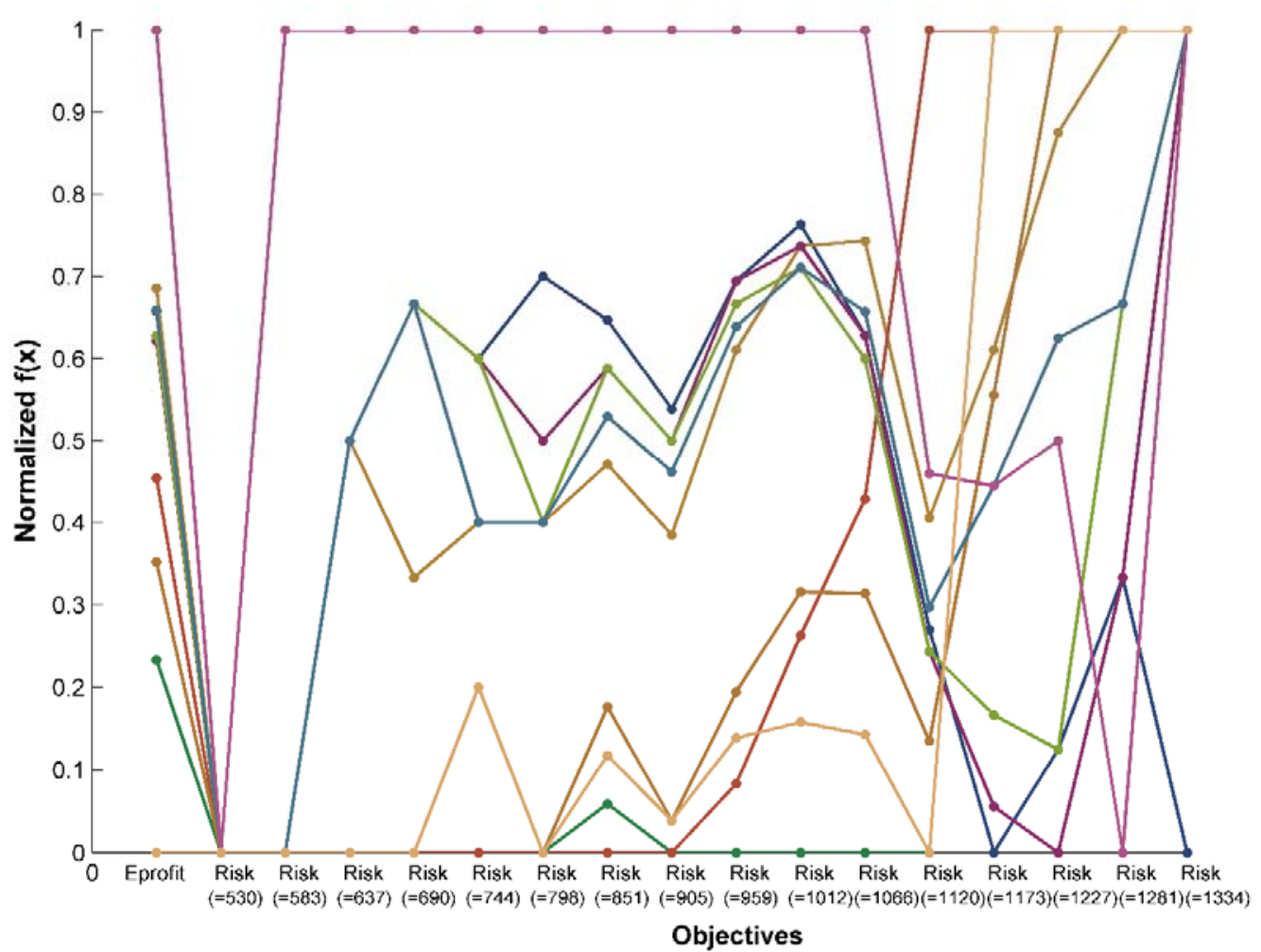

Fig.12. Cumulative probability for the solution in the reduced set of case 1 .

Notice that most of the 17 objectives behave similarly. By calculating the p-values shown in Table 5 , we can see how for 3 objectives (i.e., $\operatorname{Risk}(\Omega=530), \operatorname{Risk}(\Omega=1120)$ and $\operatorname{Risk}(\Omega=1335)$ ) a complete lack of statistical correlation is found ( $p$-values higher than 0.05 ). The highlighted values in Table 5 represent the lack of correlation among metrics. The rest of the objectives correlates each other and prove the correlation among risk metrics.

The second part of Pareto filter was next applied (order of efficiency filter) providing a deeper reduction in the pool of available solutions. Starting with the solutions obtained from the Smart filter $(k=17)$, we found non-dominated solutions in all the subsets of objectives of cardinality $k$, reducing gradually the value of $k$ until no solution satisfied the corresponding optimality level. 


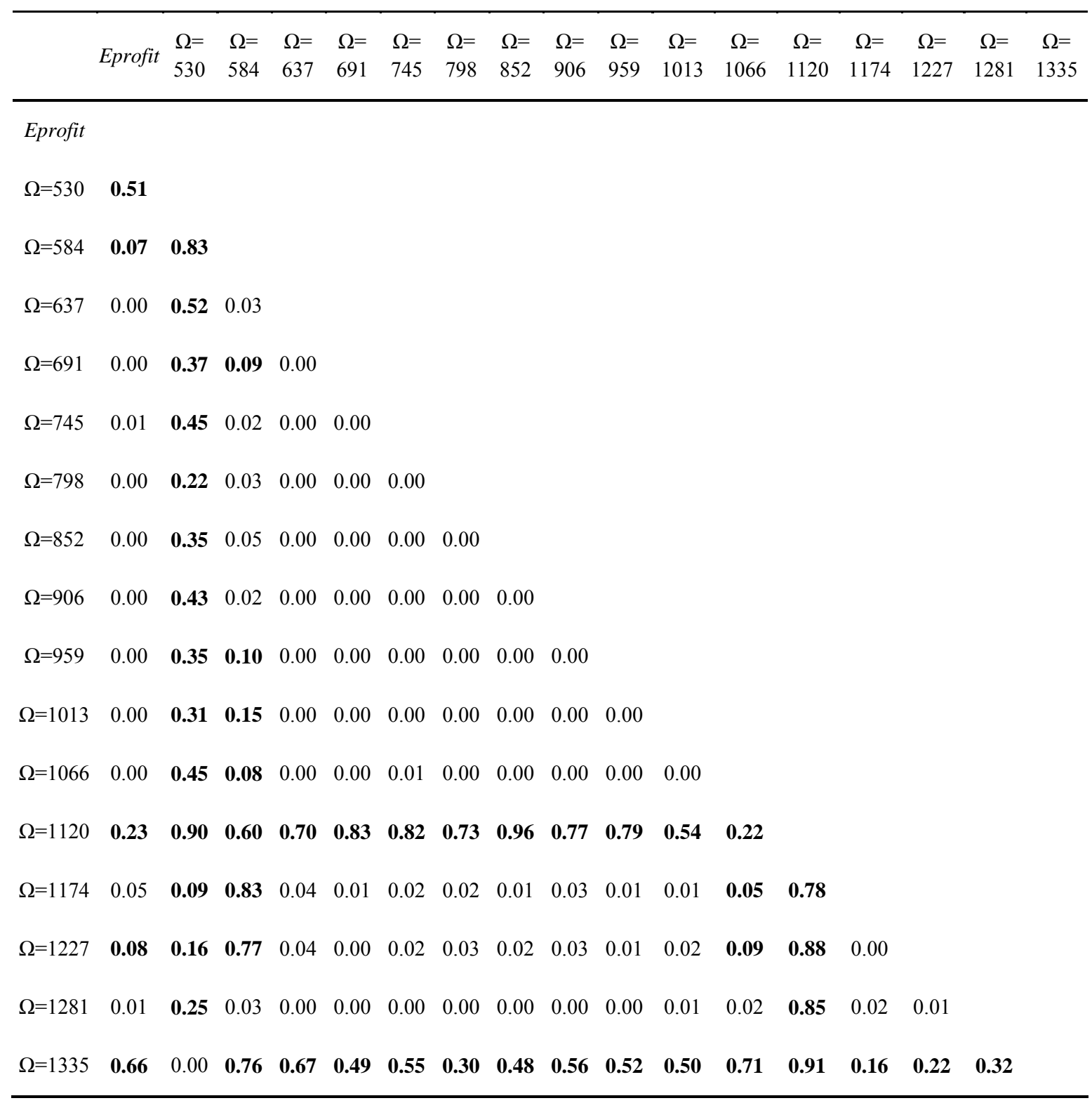

1

2 Table 6 shows the results of this filter in which reductions of 40, 60 and 90\% (from 10 to 6,4 and 1, 3 respectively) were obtained for subsets $k=16, k=15$ and $k=14$ (and $k=13$ ), respectively. For further 4 analysis $k=13$ will be omitted, since subsets for $k=14$ and $k=13$ are equal (i.e., they contain the same 5 solution).

6

7

8

Table 6. Number of solution retained in matrix $\mathrm{N}$ for each order of efficiency. 
Matrix $\boldsymbol{P}$

\begin{tabular}{lcccccc}
\hline Order of efficiency & $\mathrm{k}=17$ & $\mathrm{k}=16$ & $\mathrm{k}=15$ & $\mathrm{k}=14$ & $\mathrm{k}=13$ & $\mathrm{k}=12$ \\
$\begin{array}{l}\text { Number of } \\
\text { Solutions }\end{array}$ & 10 & 6 & 4 & 1 & 1 & 0 \\
\hline
\end{tabular}

1

2

3

Fig. 13(a, b) is analogous to Fig. 9.

(a) Lower bound

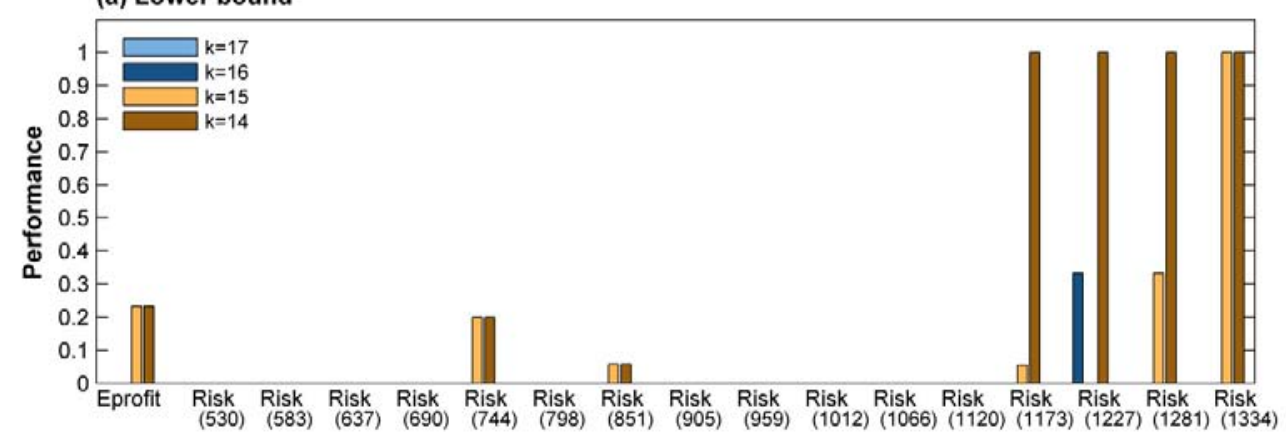

(b) Upper bound

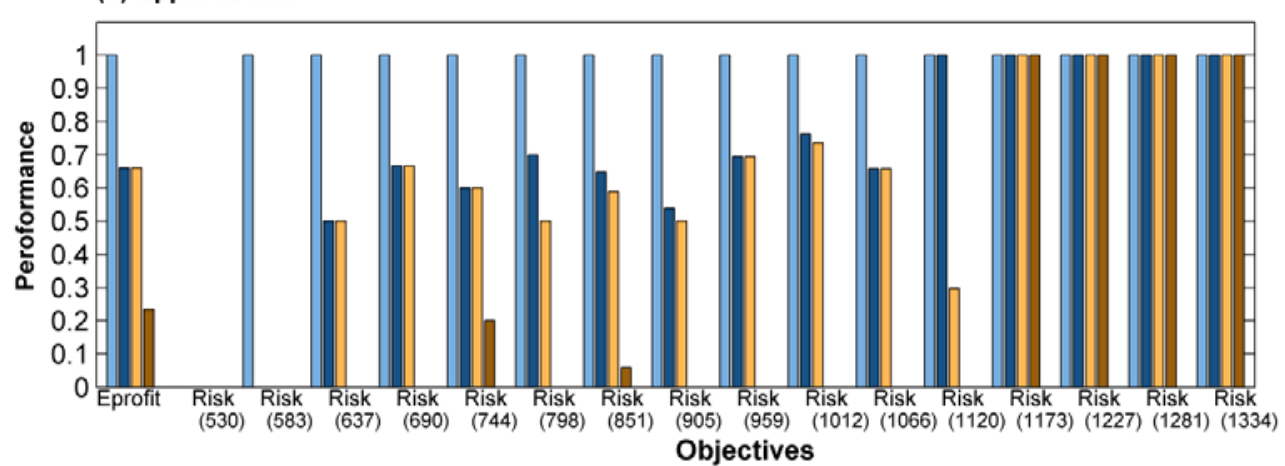

Fig. 13. Normalized bounds for solutions with efficiency of order $k$ for the second case-(a) Lower bound. (b) Upper Bound.

In Fig. 13(a) we can see that the first subset (i.e., $k=16$ ) provides an important reduction in the number of available solutions, showing similar performance than the original subset $(k=17)$. For $k=16$ only objective Risk $(=1227)$ shows a slight deviation from the best performance. This means that those solutions in subset $k=16$ has $30 \%$ less probability of achieving a profit of $\$ 1,227,000$ than the best solution in the set $k=17$. Solutions with lower orders of efficiency $(k<16)$ show a significant deterioration in their performance, specifically in the last 4 objectives (i.e., $\Omega \geq 1174$ ). Analyzing both figures we can see how the subset $k=16$ performs similarly to the subset $k=17$, but additionally 
1 eliminates solutions with poor performances (see objectives $(\Omega \leq 1066)$ in Fig. 13(b)). In view of the 2 above, we can say that the last sets of solutions (i.e., those efficient of order $k=15, k=14$ ) perform 3 better on average, but discard points with significantly better performance in some criteria. 4 Therefore, in this case the filter is stopped at $k=16$ with a reduced subset of 6 solutions, which 5 represent a total reduction of $94 \%$ in the original number of solutions (from 100 to 6).

6 Table 7 displays information on the batch plant designs associated to each solution in the reduced 7 subset, while Fig. 14 shows the cumulative distribution curves for those solutions. Table 7. Batch plant design for the reduced set of solutions in case 2.

\begin{tabular}{|c|c|c|c|c|c|c|c|c|c|}
\hline \multirow{3}{*}{ Configuration } & \multicolumn{6}{|c|}{ Second case $(k=17)$} & & & \\
\hline & \multirow{2}{*}{$\begin{array}{l}\text { Order of } \\
\text { efficiency }\end{array}$} & \multirow{2}{*}{$\begin{array}{c}\text { Eprofit } \\
\text { (M\$) }\end{array}$} & \multirow{2}{*}{$\begin{array}{c}* * \text { Demand } \\
\text { Satisfaction- } \\
(\%)\end{array}$} & \multicolumn{3}{|c|}{ Batch stage capacities $\left(\mathrm{m}^{3}\right)$} & \multicolumn{3}{|c|}{ *Storage tanks $\left(\mathrm{m}^{3}\right)$} \\
\hline & & & & $\mathrm{J} 1$ & $\mathrm{~J} 2$ & $\mathrm{~J} 3$ & $\mathrm{~J} 1$ & $\mathrm{~J} 2$ & $\mathrm{~J} 3$ \\
\hline 5 & $k=14$ & 1.073 & 76 & 1 & 0.75 & 0.5 & 0 & 5 & 10 \\
\hline 6 & $k=16$ & 1.024 & 93 & 1.2 & 0.75 & 0.5 & 0 & 0 & 5 \\
\hline 7 & $k=16$ & 1.047 & 71 & 1 & 0.75 & 0.5 & 0 & 3 & 10 \\
\hline 8 & $k=15$ & 1.028 & 92 & 1.2 & 1 & 0.5 & 0 & 5 & 5 \\
\hline 9 & $k=15$ & 1.027 & 89 & 1.2 & 1 & 0.5 & 0 & 5 & 0 \\
\hline 10 & $k=15$ & 1.024 & 85 & 1.2 & 1 & 0.5 & 0 & 5 & 0 \\
\hline
\end{tabular}




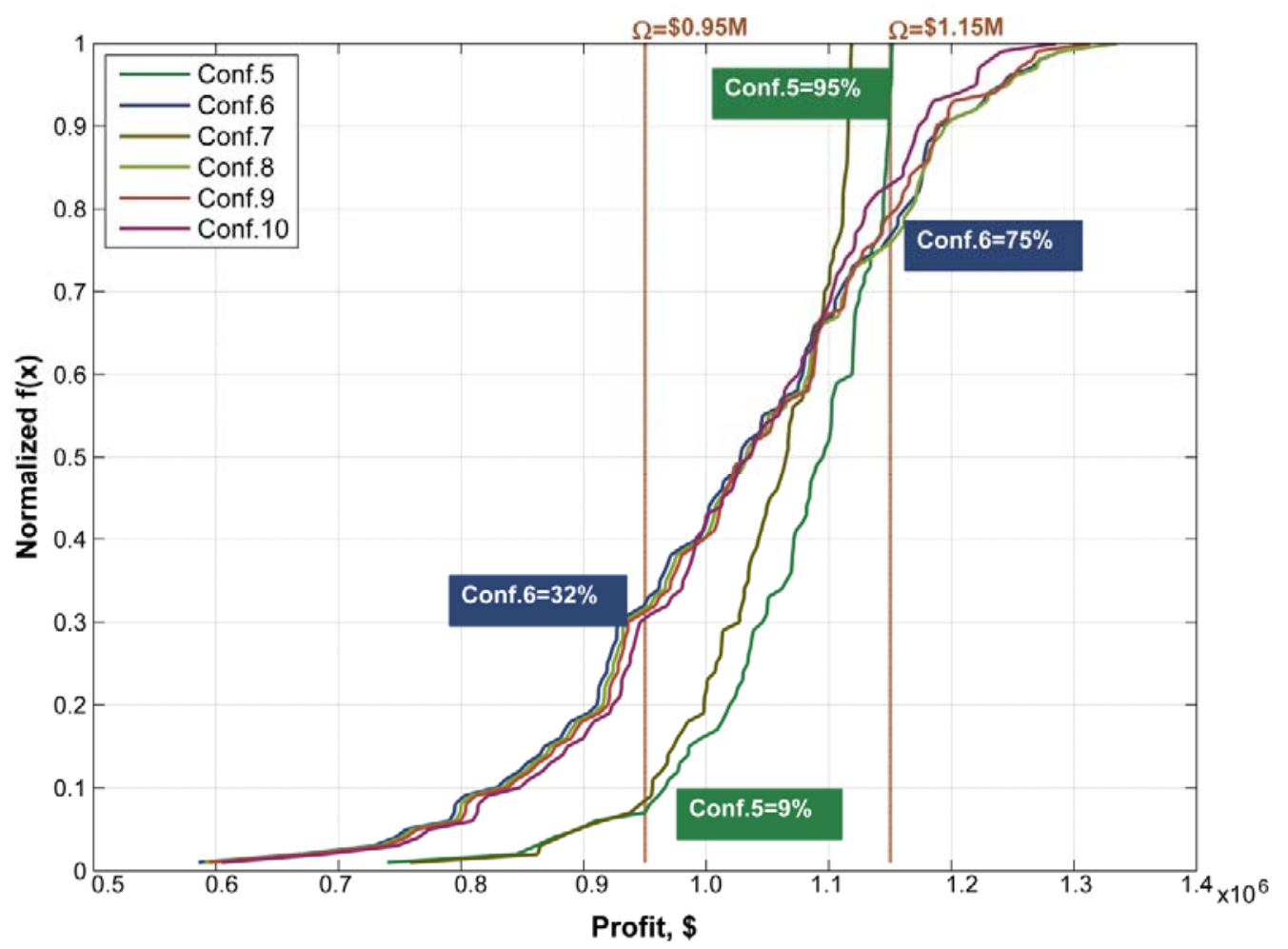

1

Fig.14. Cumulative probability curves for the solution in the reduced set of $k=16$ for case 2 .

This second case study reflects a more balanced attitude towards risk. To get insight into how the model manages risk, let us study solutions 5 and 6 . At the lower part of the profit distribution, there is a clear advantage of solution 5 over 6 , since their probabilities of profits below $\$ 0.95 \mathrm{M}$ are $9 \%$ and $32 \%$, respectively. However, for large profits (say above $\$ 1.15 \mathrm{M}$ ) those solutions behave differently achieving probabilities of $95 \%$ and $75 \%$ in configurations 5 and 6 , respectively. Notice how for lower profits solution 5 is more conservative, while for bigger profits solution 6 leads to bigger benefits.

According to Fig. 15, configuration 5 represents a very conservative solution. The most conservative solution corresponds to configuration 7 , as it provides the smallest equipment capacity at the expense of small profits (compared with solution 5). Analyzing the worst demand satisfaction level attained, configuration 6 is the best choice since its satisfaction rate is the highest one $193 \%$ in their worst scenario), while configuration 5 is the least reliable, whit a satisfaction of $76 \%$ (See Table 7). 


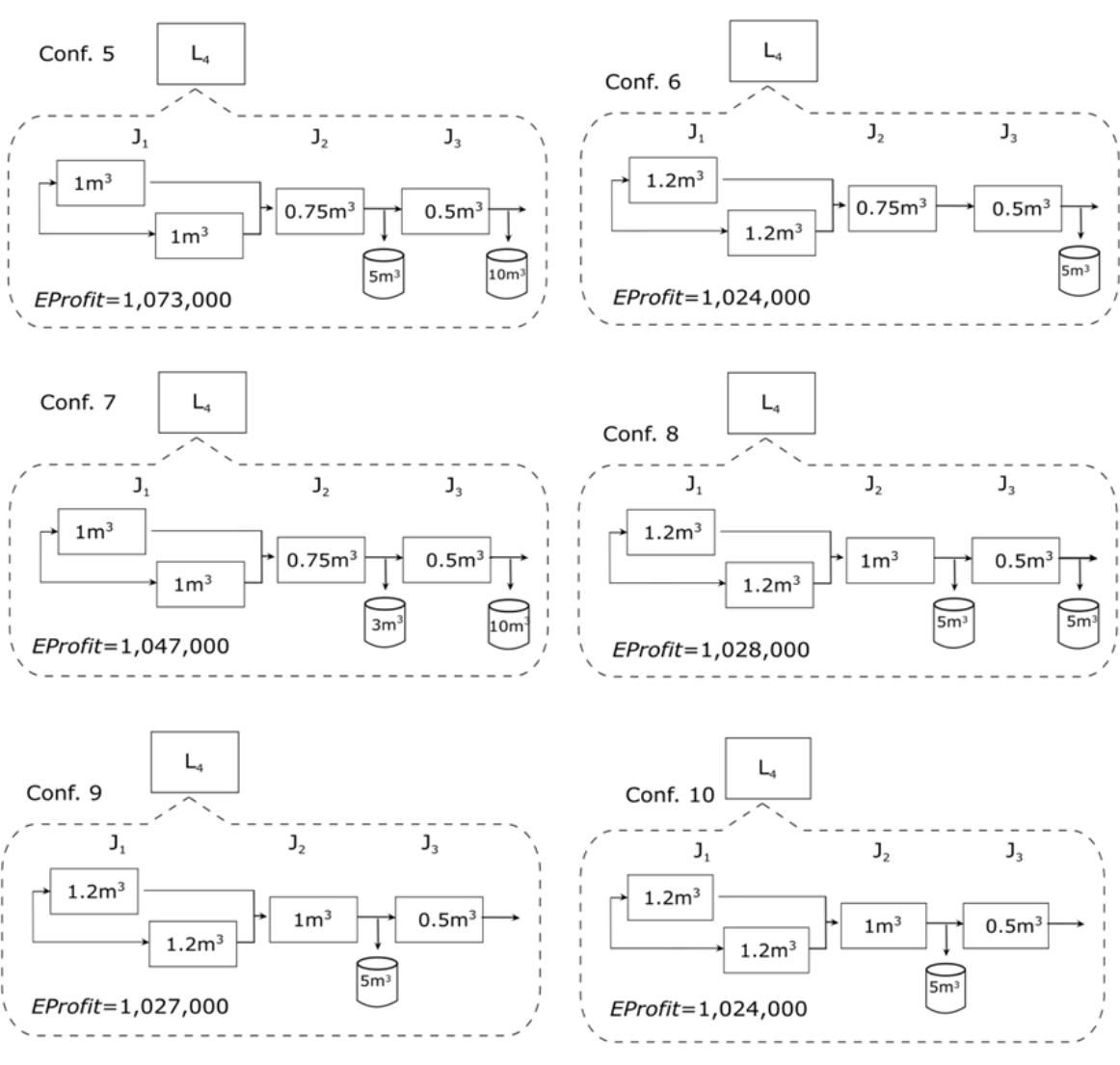

Fig. 15. Batch plant configuration scheme for the reduced set of solutions found in the second case study.

\section{Conclusions}

In this work we have proposed a systematic methodology to support risk management in optimization under uncertainty that incorporates several stochastic metrics that assess the performance of a solution considering the whole space of uncertain parameters. The proposed strategy combines optimization under uncertainty considering multiple risk metrics with a systematic approach for the selection of the most promising alternatives. The capabilities of this approach have been successfully proved using as test-bed a multi-scenario multi-objective design and planning supply chain model. Numerical results show that the proposed approach accelerates the search for supply chain design alternatives behaving in different manners in the uncertain parameters space. Furthermore, Pareto filters narrow down the number of each such alternatives, ensuring that the final design selected performs well for a wide range of economic targets.

Our tool assists decision-making by incorporating several risk metrics in the modeling framework and by avoiding subjectivity when selecting the final solution. This approach can be used in a wide variety of engineering problems in which multiple conflicting objectives and/or different performance criteria must be simultaneously considered. 


\begin{tabular}{|c|c|}
\hline Nomer & \\
\hline Abbrev & \\
\hline MOO & Multi-objective optimization \\
\hline$S C$ & Supply chain \\
\hline$S C M$ & Supply Chain Management \\
\hline MILP & Mixed integer linear programming \\
\hline PSE & Process system engineering \\
\hline$S A A$ & Sample average approximation \\
\hline$R A R$ & Risk Area Ratio \\
\hline SO & Simple Objective \\
\hline VPI & Value of Perfect Information \\
\hline Indexes & \\
\hline $\mathrm{Sp}$ & Suppliers \\
\hline $\mathrm{R}$ & Raw material \\
\hline $\mathrm{L}$ & Plants \\
\hline $\mathrm{J}$ & Batch stages \\
\hline I & Products \\
\hline M & Warehouses \\
\hline G & Customer zones \\
\hline $\mathrm{S}$ & Scenarios \\
\hline Sol & Solutions \\
\hline $\mathrm{D}$ & Parallel unit in phase \\
\hline $\mathrm{W}$ & Tanks sizes \\
\hline $\mathrm{P}$ & Batch unit discrete sizes \\
\hline Parame & \\
\hline$\Omega$ & Target value for risk metrics \\
\hline$D_{i g}$ & Demand product i for each customer zone $\mathrm{g}$ \\
\hline $\operatorname{prob}_{s}$ & Probability of occurrence for scenario e \\
\hline$\hat{f}_{l o}$ & Lower bound in normalized scale \\
\hline
\end{tabular}




\begin{tabular}{|c|c|}
\hline$\hat{f}_{\text {up }}$ & Upper bound in normalized scale \\
\hline$f_{l o}$ & Lower bound in objective value \\
\hline$f_{u p}$ & Upper bound in objective value \\
\hline$\Delta t$ & Tolerance value for Smart Pareto filter \\
\hline$f l_{r, i, j}$ & Conversion factor of raw material $r$ to produce product $I$ in batch stage $j$ \\
\hline$\Phi$ & Maximum ratio allowed between batches of consecutive stages. \\
\hline Price $_{i}$ & Selling price of product $\mathrm{i}$ \\
\hline$Q_{i, l}^{U P}$ & Upper bound for production of product $\mathrm{i}$ in plant 1. \\
\hline$Q_{i, l}^{L O}$ & Lower bound for production of product $i$ in plant 1. \\
\hline$Q_{m}^{\max }$ & Upper bound of storage capacity of warehouse $\mathrm{m}$ \\
\hline$C d_{i, m}$ & Storage cost of product $\mathrm{i}$ at storage $\mathrm{m}$ \\
\hline Cprod $_{i, l}$ & Production cost of product $i$ at plant 1 \\
\hline $\operatorname{Craw}_{s p, r}$ & Raw material acquisition cost from supplier sp and raw material $r$ \\
\hline$C t p_{i, l, m}$ & Distribution cost of product $\mathrm{i}$ among production plant 1 and storage site $\mathrm{m}$ \\
\hline$C t d_{i, m, g}$ & Distribution cost of product $\mathrm{i}$ among storage site $\mathrm{m}$ and customer zone $\mathrm{g}$ \\
\hline$S T_{i, j, l}$ & Size factor for each storage tank for contain product I at batch stage $\mathrm{j}$ in plant 1 \\
\hline$V T F_{j, l, w}$ & Discrete size $w$ for storage tanks in stage $\mathrm{j}$ of plant 1 \\
\hline \multicolumn{2}{|c|}{ Sets/subsets } \\
\hline$N_{S}$ & Set of supplier sites \\
\hline$N_{r}$ & Set of raw materials \\
\hline$N_{l}$ & Set of batch plants \\
\hline$N_{j}$ & Set of batch stages \\
\hline$N_{i}$ & Set of products \\
\hline$N_{m}$ & Set of warehouses \\
\hline$N_{g}$ & Set of customer zones \\
\hline$S$ & Set of different scenarios \\
\hline SS & Set of different solutions belonging to NSS \\
\hline
\end{tabular}




\begin{tabular}{|c|c|}
\hline Sol & Set of different solutions form model P \\
\hline RSS & Raw set of solutions \\
\hline $\bar{x}^{*}$ & Optimal set of solutions for scenario e \\
\hline $\bar{y}^{*}$ & Second stage variables in the full optimal solution \\
\hline$x x^{*}$ & Optimal solution for order of efficiency algorithm \\
\hline$s^{*}$ & Optimal set of solutions for the entire set of scenarios e \\
\hline NSS & Normalized set of solutions \\
\hline$O b$ & Objectives under analysis \\
\hline NOO & Number of objectives under analysis \\
\hline$V_{k}$ & Set of solution efficient of order $\mathrm{k}$ \\
\hline$R S$ & Set of rejected solutions \\
\hline$P$ & Solution retained after Smart Pareto filter \\
\hline M' & Set of candidate solutions \\
\hline$c^{\prime}$ & Counter set \\
\hline$c c^{\prime}$ & Counter set \\
\hline $\mathrm{K}$ & Order of efficiency \\
\hline$\Theta$ & Space of uncertain parameters \\
\hline \multicolumn{2}{|c|}{ Variables } \\
\hline$x$ & Vector of first-stage decision variables \\
\hline$\lambda$ & Random vector associated to an uncertainty behaviour \\
\hline$y$ & Vector of second-stage decision variables \\
\hline$\delta_{\Omega s}$ & Positive deviation of the profit value from the target $\Omega$ in scenario e \\
\hline Profit $_{s}$ & Profit obtained for scenario e \\
\hline$\hat{f}$ & Normalized value \\
\hline$f$ & Real objective value \\
\hline Eprofit & Objective (Expected profit) \\
\hline$Q_{s p, r, i, l, s}$ & $\begin{array}{l}\text { Material amount of raw material } r \text { send from supplier site sp to plant } 1 \text { in order to produce product } i \text { at } \\
\text { scenario } s\end{array}$ \\
\hline
\end{tabular}




\begin{tabular}{|c|c|}
\hline$B_{i, j, l, s}$ & Batch size of product $i$ at stage $j$ in plant 1 for scenario $s$ \\
\hline$N P_{j, l}$ & Number of in phase units for stage $\mathrm{j}$ in plant 1 \\
\hline$N b_{i, j, l, s}$ & Number of batches of product $\mathrm{i}$ in stage $\mathrm{j}$ of plant 1. \\
\hline$V F_{j, l, p}$ & Discrete size $\mathrm{p}$ for batch units in stage $\mathrm{j}$ of plant 1 \\
\hline$e e_{i, j, l, p, d, s}$ & Non-negative continuous variable \\
\hline$V T_{i, j, s}$ & Tank size installed for contain product i from batch stage $\mathrm{j}$ at scenario $\mathrm{s}$ \\
\hline$S S_{c}$ & Normalized solution c \\
\hline$S i z e_{i j l}$ & Size required for batch stage $\mathrm{j}$ to produce $1 \mathrm{~kg}$ of final product $\mathrm{i}$ in plant 1 \\
\hline$D R$ & Downside risk \\
\hline$W C$ & Worst case \\
\hline VaR & Value at risk \\
\hline$O V$ & Opportunity value \\
\hline Risk & Financial risk \\
\hline$V Z_{j, i, s}$ & Batch unit size of stage $j$ of plant 1 at scenario $s$ \\
\hline$f f_{i, j, l, w}$ & Continuous variable that is equal to $Q_{i l}$ if batch stage $\mathrm{j}$ is installed with tank size w \\
\hline$\rho_{i, j, l, p, n, d}$ & Auxiliary variable to skip nonlinearities \\
\hline $\mathrm{Cpl}_{l}$ & Installation cost of plant 1 \\
\hline $\mathrm{Cdep}_{m}$ & Installation cost of storage $\mathrm{m}$ \\
\hline$L C$ & Total allocation cost \\
\hline IC & Total investment cost \\
\hline$E C$ & Equipment acquisition cost \\
\hline$C_{a n}$ & Capital charge factor \\
\hline$O C_{S}$ & Total operating cost at scenario $\mathrm{s}$ \\
\hline$T C_{s}$ & Total distribution cost of scenario $\mathrm{s}$ \\
\hline $\operatorname{Ctraw}_{s p, r, i, l}$ & $\begin{array}{l}\text { Distribution cost of raw material } r \text { among supplier site sp to production plant } 1 \text { in order to produce } \\
\text { product } \mathrm{i}\end{array}$ \\
\hline TCost ${ }_{s}$ & Total cost including operating and investment cost \\
\hline Sales $_{S}$ & Total revenue obtained by selling product $i$. \\
\hline
\end{tabular}




\begin{tabular}{|ll}
\hline Binary Variables & \\
\hline$Z_{\Omega s}$ & Takes value 1 if plant 1 is allocated \\
$e x_{l}$ & Is equal to 1 if product $\mathrm{i}$ is produced in plant 1 \\
$z z_{i, l}$ & Takes value 1 if warehouse $\mathrm{m}$ is allocated for scenario $\mathrm{s}$ \\
$y y_{m, s}$ & Takes value 1 if stage $\mathrm{j}$ of plant 1 has d parallel units in phase \\
$x z_{j, l, d}$ & Determines if a tank is allocated after batch stage $\mathrm{j}$ \\
$s u_{j, l}$ & Takes value 1 if a tank of size $\mathrm{w}$ is allocated in batch stage $\mathrm{j}$ and plant 1 \\
$v t_{j, l, w}$ & Takes value 1 if a batch stage $\mathrm{j}$ is allocated to produce product $\mathrm{I}$ and with size $\mathrm{p}$ \\
$v_{j, i, p}$ &
\end{tabular}

2 Acknowledgements

3 The authors would like to thank the financial support received from the Spanish Ministry of 4 Economy and Competitiveness and the European Regional Development Fund, both funding the 5 Project ECOCIS (DPI2013-48243-C2-1-R), the Spanish "Ministerio de Ciencia y Competitividad", 6 through the project CTQ2016-77968-C3-1-P, the Generalitat de Catalunya (project 2014-SGR7 1092-CEPEiMA), the Mexican "Consejo Nacional de Ciencia y Tecnología (CONACYT)", and the 8 Pump-Priming Research Program of The University of Manchester. 


\section{References}

Ahmed S., Sahinidis N.V. 1998. "Robust Process Planning under Uncertainty”. Industrial \& Engineering Chemistry Research 37, 1883-1892.

Antipova E., Pozo C., Guillén-Gosálbez G., Boer D., Cabeza L.F., and Jiménez L. 2015. "On the Use of Filters to Facilitate the Post-Optimal Analysis of the Pareto Solutions in Multi-Objective Optimization." Computers \& Chemical Engineering.74, 48-58.

Applequist G. E., Pekny J. F., and Reklaitis G. V. 2000. "Risk and Uncertainty in Managing Chemical Manufacturing Supply Chains." Computers and Chemical Engineering. 24, 2211-2222.

Aseeri A., Gorman P., and Bagajewicz M. J. 2004. "Financial Risk Management in Offshore Oil Infrastructure Planning and Scheduling." Industrial \& Engineering Chemistry Research. 43, 3063-3072.

Aseeri A., and Bagajewicz M. J. 2004. "New Measures and Procedures to Manage Financial Risk with Applications to the Planning of Gas Commercialization in Asia." Computers and Chemical Engineering. 28, 2791-2821.

Barbaro A., and Bagajewicz M. J.. 2004. "Managing Financial Risk in Planning under Uncertainty." AIChE Journal. 50 (5), 963-89.

Ben-Tal A., El-Ghaoui L., Nemirovski A. 2009. "Robust Optimization".

Bernardo F.P., Pistikopoulos E.N., Saraiva P.M. 1999. "Integration and Computational Issues in Stochastic Design and Planning Optimization Problems". Industrial \& Engineering Chemistry Research. 38, 3056-3068

Bernardo F.P., Saraiva P.M., Pistikopoulos E.N. 2000. "Inclusion of Information Costs in Process Design Optimization under Uncertainty". Computers and Chemical Engineering 24, 1695-1701.

Bernardo F.P., Pistikopoulos E.N., Saraiva P.M. 2001. "Quality Costs and Robustness Criteria in Chemical Process Design Optimization". Computers and Chemical Engineering 25, 27-40.

Birge J.R., and Louveaux F. 2011. Introduction to Stochastic Programming.

Bolstad, B.M., Irizarry R. A., Åstrand M., and Speed T.P. 2003. “A Comparison of Normalization Metholds for High Density Oligonucleotide Array Data Based on Variance and Bias." Bioinformatics 19 (2), 185-93.

Bonfill A., Bagajewicz M.J., Espuña A., and Puigjaner L. 2004. "Risk Management in the Scheduling of Batch Plants under Uncertain Market Demand." Industrial \& Engineering Chemistry Research 43, 741-50.

Cardoso S.R., Barbosa-Póvoa A.P., and Relvas S. 2016. "Integrating Financial Risk Measures into the Design and Planning of Closed-Loop Supply Chains." Computers \& Chemical Engineering 85, 105-23.

Cheng, L., Subrahmanian E., and Westerberg A.W.. 2003. "Design and Planning under Uncertainty: Issues on Problem Formulation and Solution." Computers \& Chemical Engineering 27 (6), 781-801. 
Corsano G., Guillén-Gosálbez G., and Montagna J.M.. 2014. “Computational Methods for the Simultaneous Strategic Planning of Supply Chains and Batch Chemical Manufacturing Sites." Computers and Chemical Engineering 60, 154-171.

Corsano G., and Montagna J.M. 2011. "Mathematical Modeling for Simultaneous Design of Plants and Supply Chain in the Batch Process Industry." Computers and Chemical Engineering 35 (1), 149-64.

Das I. 1999. "A Preference Ordering among Various Pareto Optimal Alternatives." Structural Optimization 18 (1), 30-35.

Diwekar U.M., Kalagnanam J.R. 1997. "Efficient Sampling Technique for Optimization under Uncertainty”. AIChE Journal, 43 (2), 440-447.

Guillén-Gosálbez G., Grossmann I.E. 2009. "Optimal Design and Planning of Sustainable Chemical Supply Chains Under Uncertainty". AIChE Journal 55, 99-121.

Guillén-Gosálbez G., Grossmann I.E. 2010. "A global optimization strategy for the environmentally conscious design of chemical supply chains under uncertainty in the damage assessment model". Computers and Chemical Engineering. 34, 42-58.

Grossmann I.E., and Guillén-Gosálbez G. 2010. "Scope for the Application of Mathematical Programming Techniques in the Synthesis and Planning of Sustainable Processes." Computers and Chemical Engineering 34 (9), 1365-1376.

Ierapetritou M.G., Pistikopoulos E.N., Floudas C.A. 1996. “Operational Planning under Uncertainty". Computers and Chemical Engineering 20, 1499-1516.

Janak S.L., Lin X., Floudas C.A. 2007. "A new robust optimization approach for scheduling under uncertainty. II. Uncertainty with known probability distribution". Computers and Chemical Engineering 31, 171-195.

Kostin A. M., Guillén-Gosálbez G., Mele F. D., Bagajewicz M. J., and Jiménez L.. 2012. "Design and Planning of Infrastructures for Bioethanol and Sugar Production under Demand Uncertainty." Chemical Engineering Research and Design 90 (3), 359-76.

Law A.M., Kelton W.D. 2000. Simulation Modeling and Analysis, $3^{\text {rd }}$ ed. New York: McGraw Hill.

Li Z., Floudas C.A. 2014. "A Comparative Theoretical and Computational Study on Robust Counterpart Optimization: III. Improving the Quality of Robust Solutions.” Industrial \& Engineering Chemistry Research 53, 13112-13124.

Mattson C.A., and Messac A. 2003. "Concept Selection Using S-Pareto Frontiers." AIAA Journal 41 (6): 1190-98.

Mattson C.A., Mullur A.A., and Messac A.. 2004. "Smart Pareto Filter: Obtaining a Minimal Representation of Multiobjective Design Space." Engineering Optimization $36(6), 721-40$.

Plambeck E.L., Fu B.R., Robinson S.M., and Suri R.. 1996. Sample-Path Optimization of Convex Stochastic Performance Functions. Mathematical Programming 75,137-176

Pozo C., Ruíz-Femenia R., Caballero J., Guillén-Gosálbez G., and Jiménez L.. 2012a. “On the Use of Principal Component Analysis for Reducing the Number of Environmental 
Objectives in Multi-Objective Optimization: Application to the Design of Chemical Supply Chains." Chemical Engineering Science 69 (1), 146-58.

Pozo C., Guillén-Gosálbez G., Sorribas A., and Jiménez L. 2012b. "Identifying the Preferred Subset of Enzymatic Profiles in Nonlinear Kinetic Metabolic Models via Multiobjective Global Optimization and Pareto Filters.” PloS One 7 (9), e43487.

Rubinstein R.Y., and Shapiro A. 1990. "Optimization of Statistic Simulation Models by the Score Function Method." Mathematics and Computers in Simulation 32, 373-392.

Sabio N., Pozo C., Guillén-Gosálbez G., Jiménez L., Karuppiah R., Vasudevan V., Sawaya N., and Farrell J.. 2014. "Multiobjective Optimization Under Uncertainty of the Economic and Life-Cycle Environmental Performance of Industrial Processes." AIChE Journal 60, 2098-2121.

Santoso T., Ahmed S., Goetschalckx M., Shapiro A. 2005. "A stochastic programming approach for supply chain network design under uncertainty". European Journal of Operational Research 167, 96-115.

Shabbir A., and Shapiro A.. 2002. "The Sample Average Approximation Method for Stochastic Programs with Integer Recourse.” SIAM Journal on Optimization, 24

Verweij B., Ahmed S., Kleywegt A.J., Nemhauser G., Shapiro A. 2003. "The Sample Average Approximation Method Applied to Stochastic Routing Problems: A Computational Study". Computational Optimization and Application 24, 289-333.

You, F., Wassick J. M., and Grossmann I.E.. 2009. "Risk Management for a Global Supply Chain Planning Under Uncertainty Models and Algorithms.” AIChE Journal 55 (4): 931-946.

Zamarripa, M., Hjaila K., Silvente J., and Espuña A. 2014. "Tactical Management for Coordinated Supply Chains." Computers and Chemical Engineering 66: 110-23. 


\section{Appendix A}

The MILP optimizes the SC and batch plant design to maximize the expected profit considering the coordination among plants. The mathematical model is next described.

\subsection{SC network constraints.}

These constraints are related to material balances among the different nodes in the SC.

\subsubsection{Mass balances between raw material sites and production plants}

Let $\mathrm{zz}_{\mathrm{i}, 1}$ be a binary variable whose value is equal to 1 if product $\mathrm{i}$ is produced in plant 1 , and zero otherwise, and $\mathrm{Q}$ the amounts of product. Upper and lower bounds for production capacities are controlled as Eq.(A.1) shows. Additionally, Eq. (A.1) forces the total amount of product $\mathrm{i}$ in plant 1 for scenario $\mathrm{s}$ to be zero if the product is not produced in 1 .

$$
z z_{i, l} Q_{i, l}^{L O} \leq Q_{i, l, s} \leq z z_{i, l} Q_{i, l}^{U P} \quad \forall i, l, s
$$

In the same way, the use of each raw material $r$ by the plants and scenario $\mathrm{s}$ is limited according to resource availability in each raw material site sp, as shown in Eq.(A.2).

$$
\sum_{i, l} Q_{s p, r, i, l, s} \leq Q_{s p, r}^{U P} \quad \forall s p, r
$$

Also, taking into account the product allocation to a plant, each flow can be restricted according to Eq.(A.3):

$$
Q_{s p, r, i, l, s} \leq z z_{i, l} Q_{s p, r}^{U P} \quad \forall s p, r, i, l
$$

Let $f l_{r, i, l}$ be the raw material conversion factor. Eq.(A.4) defines the requirement of resource $\mathrm{r}$ in plant 1 to produce $i$.

$$
\sum_{s p=1}^{N s} Q_{s p, r, i, l, s}=f l_{r, i, l} Q_{i, l, s} \quad \forall r, i, l, s
$$

In order to avoid infeasibilities, Eq.(A.5) is included forcing the production of $i$ in plant $l$ to be zero if the plant does not exist. Therefore $e x_{l}$ is a binary variable that takes a value of 1 if plant $l$ is allocated, and zero otherwise.

$$
z z_{i, l} \leq e x_{l} \quad \forall i, l
$$

\subsubsection{Mass balances between production plants and warehouses}

The distribution links from production plants (1) to warehouses $\mathrm{m}$ in scenario $\mathrm{s}$ is constrained by Eq.(A.6): 


$$
\sum_{m=1}^{N m} Q_{i, l, m, s}=Q_{i, l, s} \quad \forall i, l, s
$$

1 A binary variable is required to control the existence of warehouses. If $y y_{m, s}$ is 1 , warehouse $m$

2 exists, and if it is zero it does not.

$$
\sum_{i, l} Q_{i, l, m, s} \leq Q_{m}^{\max } y y_{m, s} \quad \forall m, s
$$

3 Eq. (A.7) represents the constraint of received products in a warehouse $m$ according with its upper 4 capacity $\left(Q_{m}^{\max }\right)$.

$5 \quad$ 4.1.3. Mass balances between warehouses and customer zones

6 Assuming a steady-state operation (i.e., there is no stock accumulation), the total amount of product

7 i stored in warehouse $m$ has to be delivered to some customer zones g, as expressed in Eq.(A.8):

$$
\sum_{l=1}^{N l} Q_{i, l, m, s}=\sum_{g=1}^{N g} Q_{i, l, g, s} \quad \forall i, m, s
$$

8 The amount of product delivered from $\mathrm{m}$ has to be zero if the warehouse does not exist; otherwise 9 the amount of products to be stored is limited, as shown in Eq. (A.9).

$$
\sum_{i, g} Q_{i, m, g, s} \leq Q_{m}^{\max } y y_{m, s} \quad \forall m, s
$$

10 Disregarding the installation or not of warehouses the demands have to be fulfilled, as shown in Eq. 11 (A.10).

$$
D_{i, g}=\sum_{m=1}^{N m} \sum_{l} Q_{i, l, m, s} \quad \forall i, s
$$

12

\section{A.2. Production plant design equations}

\section{A.2.1. Batch units}

Considering the common assumptions for multiproduct batch plant design and the general batch process literature, the batch unit size of stage $\mathrm{j}$ of plant $1, V Z_{j, l, s}$, is computed through a sizing equation (Eq.(A.11)) which is applied for each product i as follows:

$$
V Z_{j, l, s} \geq \frac{\text { Size }_{i, j, l} B_{i, j, l, s}}{N P_{j, l}} \quad \forall i, j, l, s
$$


1 where $\operatorname{Size}_{i, j, l}$ is the size factor (the size required at stage $\mathrm{j}$ to produce $1 \mathrm{~kg}$ of final product $\mathrm{i}$ ), $B_{i, j, l, s}$

2 is the batch size of product $\mathrm{i}$ at stage $\mathrm{j}$ in plant 1 at any scenario $\mathrm{s}$, and $N P_{j, l}$ is the number of in

3 phase units for stage $\mathrm{j}$ in plant 1 . Let $N b_{i, j, l, s}$ be the number of batches of product $\mathrm{i}$ in stage $\mathrm{j}$ of plant

41 and scenario $\mathrm{s}$. The amount of product i produced in plant 1 is defined by Eq.(A.12)

$$
Q_{i, l, s}=N b_{i, j, l, s} B_{i, j, l, s} \quad \forall i, j, l, s
$$

5 By combining Eq. (A.11) and Eq. (A.12), the following constraint is obtained

$$
N b_{i, j, l, s} \geq \frac{\operatorname{Size}_{i, j, l} Q_{i, l, s}}{V Z_{j, l, s} N P_{j, l}} \quad \forall i, j, l, s
$$

6 In order to formulate the problem as an MILP, non-linear constraints are avoided by rewriting the 7 previous design equation (Eq.(A.13)) in the following manner (Eq.(A.14)). Let $x z_{j, l, d}$ be the binary 8 variable that takes a value of 1 if stage $\mathrm{j}$ of plant 1 has $d$ parallel units in phase, and zero otherwise, 9 then we have:

$$
\begin{array}{cc}
N P_{j, l}=\sum_{d=1}^{N P_{j, l}^{U P}} d x z_{j, l, d} & \forall j, l \\
\sum_{d=1}^{N P_{j l}^{U P}} x z_{j, l, d}=e x_{l} & \forall j, l
\end{array}
$$

where $N P_{i, j}^{U P}$ represents the total available units in phase for stage $\mathrm{j}$ in plant 1 . Eq. (A.15) states that at least one unit per stage must exist if plant 1 is allocated.

The size for unit $\mathrm{j}$ of plant $1, V Z_{j, l, s}$, considering a set of available discrete sizes $\mathrm{p}$, is given by Eq. (A.16) while Eq.(A.17) defines if a plant will be allocated (or not) as well as the discrete size of the plant:

$$
\begin{aligned}
V Z_{j, l, s}=\sum_{p=1}^{P_{j, l}} V Z_{j, l, p, s} V F_{j, l, p} & \forall j, l, s \\
\sum_{p \in S V_{j l}} V Z_{j, l, p, s}=e x_{l} & \forall j, l, s
\end{aligned}
$$

15 Substituting Eq. (A.17) for Eq. (A.13) under $\mathrm{NP}_{\mathrm{j}, 1}$ definition

$$
N b_{i, j, l, s} \geq \sum_{p, d} \frac{\text { Size }_{i, j, l} Q_{i, l, s}}{V F_{j, l, p} d} v_{j, l, p} x z_{j, l, d} \quad \forall i, j, l, s
$$


1 A new nonnegative continuous variable, $e e_{i, j, l, p, d, s}$, is defined, since constraint Eq. (A.18) is non-

2 linear because of the product between $Q_{i, l, s}, v_{j, l, p}$ and $x z_{j, l, d}$.

$$
e e_{i, j, l, p, d, s}=\left\{\begin{array}{ccc}
Q_{i, l, s} & \text { if } & v_{j, l, p} \text { and } x z_{j, l, d}=1 \\
& 0 & \text { otherwise }
\end{array}\right\} \quad \forall i, j, l, p, d, s
$$

3

4 Therefore, the following linear constraints (Eqs.(A.20-A.23)) are used to represent Eq. (A.18):

$$
\begin{array}{cc}
N b_{i, j, l, s} \geq \sum_{p, d} \frac{\operatorname{Size}_{i, j, l}}{V F_{j, l, p} d} e e_{i, j, l, p, d, s} & \forall i, j, l, s \\
\sum_{d} e e_{i, j, l, p, d, s} \leq Q_{i, l}^{U P} v_{j, l, p} & \forall i, j, l, p, s \\
\sum_{p} e e_{i, j, l, p, d, s} \leq Q_{i, l}^{U P} x Z_{j, l, p} & \forall i, j, l, d, s \\
Q_{i, l, s}=\sum_{p, d} e e_{i, j, l, p, d, s} & \forall i, j, l, s
\end{array}
$$

\section{$5 \quad$ A.2.2. Intermediate storage}

6 For $\mathrm{N}_{\mathrm{j} 1}$ batch stages there exist, at most, $\mathrm{N}_{\mathrm{j} 1-1}$ possible positions for storage tanks to be allocated 7 between two consecutive batch stages. Therefore, an upper bound for the storage vessels can be 8 defined by Eqs.(A.24-A.25)

$$
\begin{array}{lr}
V T_{j, i, s} \geq 2 S T_{i, j, l} B_{i, j, l, s} s u_{j, l} & \forall i, l, s, j=1,2, \ldots, N_{j, l-1} \\
V T_{j, i, s} \geq 2 S T_{i, j, l} B_{i, j+1, l, s} s u_{j, l} & \forall i, l, s, j=1,2, \ldots, N_{j, l-1}
\end{array}
$$

9 where $V T_{j, i, s}$ represents the tank size, $S T_{i, j, l}$ is the size factor for each storage tank and $s u_{j, l}$ is a 10 binary variable that determines if a tank is allocated after batch stage $j$ with the value of 1 , and zero 11 otherwise. Using Eq.(A.11) in Eq.(A.13) and Eq.(A.25), the storage constraints are rewritten as Eq.(A.26-A.27):

$$
\begin{array}{ll}
N b_{i, j, l, s} \geq 2 \frac{S T_{i, j, l} Q_{i, l, s}}{V T_{j, l, s}} s u_{j, l} & \forall i, l, s, j=1,2, \ldots, N_{j l-1} \\
N b_{i, j+1, l, s} \geq 2 \frac{S T_{i, j, l} Q_{i, l, s}}{V T_{j, l, s}} s u_{j, l} & \forall i, l, s, j=1,2, \ldots, N_{j l-1}
\end{array}
$$


Again, in order to avoid nonlinearities, a set of available discrete sizes for the tank allocated after stage $\mathrm{j}, \mathrm{STF}_{\mathrm{j}, 1}=\left\{V T F_{j, l, 1}, V T F_{j, l, 2}, \ldots, V T F_{j, l, w}\right\}$, is selected. Let $v t_{j, l, w}$ be the binary variable that takes a value 1 if a tank of size $w$ is allocated in position $\mathrm{j}$ and zero otherwise. The first tank size of the set, $V T F_{j l 1}$, is equal to zero to represent "no tank allocation". Then, Eq. (A.26) and Eq. (A.27) are rewritten as Eq.(A.28-A.30):

$$
\begin{array}{cr}
N b_{i, j, l, s} \geq 2 \sum_{w \neq 1} \frac{S T_{i, j, l} Q_{i, l, s}}{V T F_{j, l, w}} v t_{j l w} & \forall i, l, s, j=1,2, \ldots, N_{j l-1} \\
N b_{i, j+1, l s} \geq 2 \sum_{w \neq 1} \frac{S T_{i, j, l} Q_{i, l, s}}{V T F_{j, l, w}} v t_{j, l, w} & \forall i, l, s, j=1,2, \ldots, N_{j l-1} \\
\sum_{w} v t_{j, l, w}=e x_{l} & \forall l, j=1,2, \ldots, N_{j l-1}
\end{array}
$$

Eq. (A.30) states that if plant 1 exists, then only one discrete size for a tank after stage $\mathrm{j}$ has to be selected. Using the continuous variable $f f_{i, j, l, w}=Q_{i, l} v t_{j, l, w}$, Eq.(A.28) and Eq.(A.29) become linear and give rise to Eq.(A.31-A.32) using the constraints represented in Eq.(A.33-A.34).

$$
\begin{array}{cc}
N b_{i, j, l, s} \geq 2 \sum_{w \neq 1} \frac{S T_{i, j, l}}{V T F_{j, l, w}} f f_{i, j, l, w} & \forall i, l, s, j=1,2, \ldots, N_{j, l-1} \\
N b_{i, j+1, l s} \geq 2 \sum_{w \neq 1} \frac{S T_{i, j, l}}{V T F_{j, l, w}} f f_{i, j, l, w} & \forall i, l, s, j=1,2, \ldots, N_{j, l-1} \\
f f_{i, j, l, w} \leq Q_{i, l}^{U P} v t_{j, l, w} & \forall i, l, j=1,2, \ldots, N_{j, l-1}, w \\
Q_{i, l, s}=\sum_{w} f f_{i, j, l, w} & \forall i, l, s, j=1,2, \ldots, N_{j, l-1}
\end{array}
$$

If the storage tank does not exist between two consecutive batch stages, then the number of batches must be equal for both of them. Else, the bounds for the ratio between the numbers of batches of consecutive stages can be stated by Corsano et al., 2011 and calculated as in Eq.(A.35):

$$
\begin{aligned}
N b_{i, j, l, s} & +\left(\frac{1}{\phi}-1\right) \sum_{w \neq 1} v t_{j, l, w} \leq \frac{N b_{i, j+1, l, s}}{N b_{i j l s}} \quad \forall i, l, s, j=1,2, \ldots, N_{j, l-1} \\
& \leq 1+(\phi-1) \times \sum_{w \neq 1} v t_{j, l, w}
\end{aligned}
$$

where $\phi$ is a constant value corresponding to the maximum ratio allowed between the number of batches of consecutive stages.

\section{A.3. Objective Function}


1 The investment cost considers the batch units and storage tanks cost. In this work, they are selected

2 from a set of available discrete sizes. Then, equipment cost (EC) is given by Eq. (A.36)

$$
\begin{aligned}
E C=\sum_{l} \sum_{j} \sum_{p} \sum_{n} \sum_{d} \alpha_{j, l} V F_{j, l, p}^{\left(\beta_{j, l}\right)} v_{j, l, p} n x_{j, l, n} d x z_{j, l, d} \\
+\sum_{l} \sum_{j} \sum_{w} \bar{\alpha}_{j, l} V T F_{j, l, w}^{\bar{\beta}_{j, l}} v t_{j l w}
\end{aligned}
$$
Then the equipment cost can be rewritten as in Eq.(A.39).

$$
\begin{aligned}
E C=\sum_{l} \sum_{j} \sum_{p} \sum_{n} \sum_{d} \alpha_{j, l} n d V F_{j, l, p}^{\left(\beta_{j, l}\right)} \rho_{i, j, l, p, n, d} & \\
& +\sum_{l} \sum_{j} \sum_{w} \bar{\alpha}_{j, l} V T F_{j, l, w}^{\bar{\beta}_{j l}} v t_{j, l, w}
\end{aligned} \quad \forall j, l
$$

8 A fixed investment cost is considered if production plants and warehouses are installed. Then, the 9 allocation cost (LC) is given by Eq.(A.40), in which $C p l_{l}$ and $C d e p_{m}$ are the installation cost

Therefore, the total investment cost is described in Eq.(A.41).

$$
I C=C_{a n}(E C+L C)
$$

The operating cost, including raw material acquisition, storage, and production cost are considered through the following expression (Eq.(A.42)).

$$
\begin{gathered}
o C_{s}=\sum_{s p} \sum_{r} \sum_{i} \sum_{l} \operatorname{Craw}_{s p, r} Q_{s p, r, i, l, s}+\sum_{i} \sum_{l} \sum_{m} C d_{i, m} Q_{i, l, m, s} \\
+\sum_{i} \sum_{l} \operatorname{Cprod}_{i, l} Q_{i, l, s}
\end{gathered}
$$

$\operatorname{Craw}_{s p, r}, C d_{i, m}$, and $C \operatorname{Crod}_{i, i}$ are the associated costs for raw material acquisition, storage, and production cost, respectively. The $Q$ amounts are expressed in kg per time horizon, therefore the cost parameters are given in $\$ / \mathrm{kg}$. 
1 The distribution costs at the entire supply chain are also considered in this model. Those costs are

2 represented through the Eq. (A.43).

$$
\begin{gathered}
T C_{s}=\sum_{s p} \sum_{r} \sum_{i} \sum_{l} \operatorname{Ctraw}_{s p, r, l} Q_{s p, r, i, l, s}+\sum_{m} \sum_{i} \sum_{l} \operatorname{Ctp}_{i, l, m} Q_{i, l, m, s} \\
+\sum_{i} \sum_{m} \sum_{k} C t d_{i, m, k} Q_{i, m, k, s}
\end{gathered} \forall s
$$

3 In Eq.(A.43), $C t r a w_{s p, r, l}, C t p_{i, l, m}$, and $C t d_{i, m, k}$ are cost coefficients that depend on the product

4 transported and the covered distance. Eq.(A.44) summarize the total cost for each scenario.

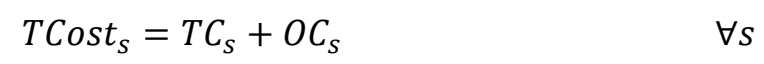

5 Eq.(A.45) describes the economic revenue of selling the final product at each scenario. Where 6 Price $_{i}$ is the selling price of product $\mathrm{i}$ in $\$ / \mathrm{kg}$. The profit at each scenario is obtained through the 7 difference among economic revenue and associated costs at each scenario realization as represented 8 in Eq.(A.46).

$$
\begin{array}{cc}
\text { Sales }_{S}=\sum_{i} \sum_{m} \sum_{k} Q_{i, m, k, S} \text { Price }_{i} & \forall s \\
\text { PROFIT }_{S}=\text { Sales }_{S}-\text { TCost }_{s} & \forall s
\end{array}
$$

9 In Eq.(A.47) the total expected profit is described, in which the associated probability of each 10 scenario is taken into account. Additionally, costs which don't depend on the scenario realization 11 are considered in this equation, denoted as IC.

$$
\text { EProfit }=\left(\sum_{s} \text { PROFIT }_{S} \text { Prob }_{S}\right)-I C
$$

The objective function to be maximized is as follows.

$$
\max \{\text { EProfit }\}
$$

13 For more details on the model, authors invite the readers to check Corsano et al., 2011 


\section{Appendix B}

2 Table B.1. Raw material costs.

\begin{tabular}{|c|c|c|c|c|c|c|c|c|}
\hline & \multicolumn{5}{|c|}{ Distribution Cost $\left(\$ \mathrm{~kg}^{-1}\right)$} & \multicolumn{3}{|c|}{ Procurement cost $\left(\$ \mathrm{~kg}^{-1}\right)$} \\
\hline & $1_{1}$ & $1_{2}$ & $1_{3}$ & $1_{4}$ & $1_{5}$ & $\mathrm{r}_{1}$ & $\mathrm{r}_{2}$ & $r_{3}$ \\
\hline $\mathrm{s}_{1}$ & 0.02 & 0.1 & 0.08 & 0.06 & 0.08 & 0.02 & 0.02 & 0.01 \\
\hline $\mathrm{S}_{2}$ & 0.14 & 0.12 & 0.14 & 0.02 & 0.06 & 0.02 & 0.01 & 0.02 \\
\hline
\end{tabular}

3

4

5 Table B.2.Product demand (Ton).

\begin{tabular}{lllll}
\hline & $\mathrm{i}_{1}$ & $\mathrm{i}_{2}$ & $\mathrm{i}_{3}$ & $\mathrm{i}_{4}$ \\
\hline $\mathrm{g}_{1}$ & 150 & 130 & 150 & 100 \\
$\mathrm{~g}_{2}$ & 100 & 120 & 150 & 100 \\
$\mathrm{~g}_{3}$ & 115 & 130 & 150 & 120 \\
\hline
\end{tabular}

6

7 Table B.3. Product distribution cost form plant to warehouse.

Distribution Cost $\left(\$ \mathrm{~kg}^{-1}\right)$

\begin{tabular}{|c|c|c|c|c|c|c|c|c|c|c|c|c|}
\hline & \multicolumn{4}{|c|}{$\mathrm{m}_{1}$} & \multicolumn{4}{|c|}{$\mathrm{m}_{2}$} & \multicolumn{4}{|c|}{$\mathrm{m}_{3}$} \\
\hline & $\mathrm{i}_{1}$ & $\mathrm{i}_{2}$ & $\mathrm{i}_{3}$ & $\mathrm{i}_{4}$ & $\mathrm{i}_{1}$ & $\mathrm{i}_{2}$ & $\mathrm{i}_{3}$ & $\mathrm{i}_{4}$ & $\mathrm{i}_{1}$ & $\mathrm{i}_{2}$ & $\mathrm{i}_{3}$ & $\mathrm{i}_{4}$ \\
\hline $1_{1}$ & 0.1 & 0.17 & 0.05 & 0.05 & 0.2 & 0.1 & 0.15 & 0.15 & 0.23 & 0.16 & 0.11 & 0.11 \\
\hline $1_{2}$ & 0.2 & 0.19 & 0.25 & 0.25 & 0.19 & 0.18 & 0.35 & 0.35 & 0.18 & 0.19 & 0.15 & 0.15 \\
\hline $1_{3}$ & 0.2 & 0.18 & 0.25 & 0.25 & 0.18 & 0.15 & 0.25 & 0.25 & 0.15 & 0.08 & 0.15 & 0.18 \\
\hline $1_{4}$ & 0.05 & 0.1 & 0.2 & 0.15 & 0.15 & 0.11 & 0.2 & 0.2 & 0.1 & 0.15 & 0.15 & 0.05 \\
\hline $1_{5}$ & 0.2 & 0.18 & 0.25 & 0.25 & 0.2 & 0.15 & 0.25 & 0.25 & 0.15 & 0.15 & 0.08 & 0.08 \\
\hline
\end{tabular}

8

9 
1 Table B.4. Product distribution cost $\left(\$ \mathrm{~kg}^{-1}\right)$ from warehouse to customer.

\begin{tabular}{cccc}
\hline & $\mathrm{m}_{1}$ & $\mathrm{~m}_{2}$ & $\mathrm{~m}_{3}$ \\
\hline $\mathrm{g}_{1}$ & 0.08 & 0.09 & 0.09 \\
$\mathrm{~g}_{2}$ & 0.07 & 0.09 & 0.08 \\
$\mathrm{~g}_{3}$ & 0.06 & 0.07 & 0.05 \\
\hline
\end{tabular}

2

3 Table B.5. Batch parameters.

Batch parameters

\begin{tabular}{|c|c|c|c|c|c|c|c|c|c|c|c|c|c|c|}
\hline & \multicolumn{3}{|c|}{ Size factors } & \multicolumn{3}{|c|}{ Operating time $(\mathrm{h})$} & \multicolumn{3}{|c|}{$\begin{array}{l}\text { Raw material factor } \\
\text { conversion }\end{array}$} & \multicolumn{5}{|c|}{ Production Cost $\left(\$ \mathrm{~kg}^{-1}\right)$} \\
\hline & $\mathrm{j}_{1}$ & $\mathrm{j}_{2}$ & $\mathrm{j}_{3}$ & $\mathrm{j}_{1}$ & $\mathrm{j}_{2}$ & $\mathrm{j}_{3}$ & $\mathrm{r}_{1}$ & $\mathrm{r}_{2}$ & $r_{3}$ & $1_{1}$ & $1_{2}$ & $1_{3}$ & $1_{4}$ & $1_{5}$ \\
\hline$i_{1}$ & 0.9 & 0.6 & 0.4 & 14 & 5 & 7 & 0.8 & 0.5 & 0.7 & 0.12 & 0.18 & 0.12 & 0.06 & 0.12 \\
\hline $\mathrm{i}_{2}$ & 0.6 & 0.5 & 0.4 & 12 & 6 & 4 & 0.6 & 0.8 & 0.8 & 0.08 & 0.16 & 0.06 & 0.12 & 0.10 \\
\hline $\mathrm{i}_{3}$ & 0.7 & 0.5 & 0.4 & 16 & 8 & 5 & 0.4 & 0.5 & 0.5 & 0.12 & 0.14 & 0.14 & 0.08 & 0.14 \\
\hline $\mathrm{i}_{4}$ & 0.8 & 0.6 & 0.4 & 10 & 4 & 5 & 0.5 & 0.5 & 0.5 & 0.14 & 0.08 & 0.14 & 0.04 & 0.12 \\
\hline
\end{tabular}

4

5

6

7

8

9

10

11

12

13

14

15 
Table B.6. Batch costs.

Batch investment cost

\begin{tabular}{|c|c|c|c|c|c|c|c|c|c|c|c|c|c|}
\hline & \multicolumn{5}{|c|}{ Unit cost coefficient $\alpha_{j i}$ (annualized) } & \multicolumn{3}{|c|}{$\begin{array}{c}\text { Raw material factor } \\
\text { conversion }\end{array}$} & \multicolumn{5}{|c|}{ Production Cost $\left(\$ \mathrm{~kg}^{-1}\right)$} \\
\hline & $1_{1}$ & $1_{2}$ & $1_{3}$ & $1_{4}$ & $1_{5}$ & $\mathrm{r}_{1}$ & $\mathrm{r}_{2}$ & $r_{3}$ & $1_{1}$ & $1_{2}$ & $1_{3}$ & $1_{4}$ & $1_{5}$ \\
\hline $\mathrm{j}_{1}$ & 1620 & 2430 & 1350 & 1350 & 1890 & 0.8 & 0.5 & 0.7 & 0.12 & 0.18 & 0.12 & 0.06 & 0.12 \\
\hline $\mathrm{j}_{2}$ & 2160 & 1620 & 2160 & 1620 & 1890 & 0.6 & 0.8 & 0.8 & 0.08 & 0.16 & 0.06 & 0.12 & 0.1 \\
\hline $\mathrm{j}_{3}$ & 1890 & 2700 & 1890 & 1890 & 2430 & 0.4 & 0.5 & 0.5 & 0.12 & 0.14 & 0.14 & 0.08 & 0.14 \\
\hline Tanks & 500 & 500 & 500 & 500 & 500 & 0.5 & 0.5 & 0.5 & 0.14 & 0.08 & 0.14 & 0.04 & 0.12 \\
\hline
\end{tabular}

2

3 


\section{Appendix C}

The number of scenarios (i.e., sample size) required to ensure a good estimation of the "real" values in the domain of uncertain parameters is a critical issue in any multiscenario problem. In this regard, the method proposed by Law and Kelton (2000), represents a promising alternative and it is completely applicable to any stochastic programming model. This approach relies on solving the stochastic model iteratively for an increasing number of scenarios until a given relative error $\gamma$ is satisfied for a confidence level of $100(1-\alpha \alpha) \%$. In the context of our problem, this method comprises the following steps:

1. Define an initial number of scenarios $\mathrm{ns}_{0}$, (as $|\mathrm{S}|=\mathrm{s}=\mathrm{ns}_{0}$ ) where $\mathrm{s}$ will be updated dynamically during the execution of the algorithm.

2. Solve the specific stochastic model with $|\mathrm{S}|=\mathrm{s}$ scenarios

3. Compute the confidence interval half-length $\vartheta(s, a a)$ for the mean value of the values in each scenario using Eq.B1.

$$
\vartheta(s, a \alpha)=t_{n-1, \frac{1-a \alpha}{2}} \sqrt{\frac{\operatorname{Var}^{2}(s)}{s}}
$$

Where $\operatorname{Var}^{2}(s)$ is the sample variance, and $t_{n-1, \frac{1-a \alpha}{2}}$ is the critical point of the $\mathrm{t}$ distribution.

4. If $\frac{\delta(s, \alpha)}{\mid \text { Evalue } \mid} \leq \frac{\gamma}{1-\gamma}$, then stop (i.e., the expected value of the discrete distribution is a valid estimator of the mean of the universe for the relative error and confidence interval defined beforehand). Otherwise, make $s=s+1$ and go to Step 2 .

More details about this procedure can be found in the original work by Law and Kelton (2000). 